\title{
Shellings and sheddings induced by collapses*
}

\author{
Thomas Magnard \\ Université Paris-Est, LIGM, CNRS, ENPC, ESIEE Paris, UPEM, Marne-la-Vallée, France \\ Michael Skotnica \\ Department of Applied Mathematics, Charles University, Prague, Czech Republic \\ Martin Tancer \\ Department of Applied Mathematics, Charles University, Prague, Czech Republic
}

\begin{abstract}
We say that a pure simplicial complex $\mathbf{K}$ of dimension $d$ satisfies the removal-collapsibility condition if $\mathbf{K}$ is either empty or $\mathbf{K}$ becomes collapsible after removing $\tilde{\beta}_{d}\left(\mathbf{K} ; \mathbb{Z}_{2}\right)$ facets, where $\tilde{\beta}_{d}\left(\mathbf{K} ; \mathbb{Z}_{2}\right)$ denotes the $d$ th reduced Betti number.

In this paper, we show that if the link of each face of a pure simplicial complex $\mathbf{K}$ (including the link of the empty face which is the whole $\mathbf{K}$ ) satisfy the removal-collapsibility condition, then the second barycentric subdivision of $\mathbf{K}$ is vertex decomposable and in particular shellable. This is a higher dimensional generalization of a result of Hachimori, who proved that if the link of each vertex of a pure 2-dimensional simplicial complex $\mathbf{K}$ is connected, and $\mathbf{K}$ becomes simplicially collapsible after removing $\tilde{\chi}(\mathbf{K})$ facets, where $\tilde{\chi}(\mathbf{K})$ denotes the reduced Euler characteristic, then the second barycentric subdivision of $\mathbf{K}$ is shellable.

For the proof, we introduce a new variant of decomposability of a simplicial complex, stronger than vertex decomposability, which we call star decomposability. This notion may be of independent interest.
\end{abstract}

\section{Introduction}

Shellability and collapsibility (to be defined later) are two widely used approaches for combinatorial decomposition of a simplicial complex. They are similar in spirit, yet there are important differences among those two notions. There are shellable complexes homotopy equivalent to a wedge of spheres, whereas no non-trivial wedge can be collapsible. On the other hand, two triangles sharing a vertex provide an example of a collapsible complex that is not shellable. Yet in some important cases, one can relate these two notions.

The easy direction is that shellability implies collapsibility whenever the complex is contractible (in fact, whenever the complex has trivial homology). We will focus here on a more interesting direction: when collapsibility implies shellability?

In this spirit, Hachimori Hac08] proved that for a pure 2-dimensional simplicial complex $\mathbf{K}$, the following statements are equivalent:

(i) The complex $\mathbf{K}$ has a shellable subdivision.

(ii) The second barycentric subdivision $\mathrm{sd}^{2} \mathbf{K}$ is shellable.

\footnotetext{
${ }^{*}$ This work was supported by the grant no. 19-04113Y of the Czech Science Foundation (GAČR). T.M. is supported by grant ANR-17-CE40-0033 of the French National Research Agency ANR (SoS project). M.T. is partially supported by Charles University project UNCE/SCI/004.
} 
(iii) The link of each vertex of $\mathbf{K}$ is connected and $\mathbf{K}$ becomes collapsible after removing $\tilde{\chi}(\mathbf{K})$ facets where $\tilde{\chi}$ denotes the reduced Euler characteristic.

As Hachimori points out, one cannot expect that such an equivalence would be achievable in higher dimensions. Namely, the implication (i) $\Rightarrow$ (ii) cannot hold in higher dimensions due to the examples by Lickorish [Lic91]. However, we will show that it is possible to generalize the interesting implication (iii) $\Rightarrow$ (ii). The equivalence of (iii) and (ii) was one of the important steps in a recent proof of NP-hardness of recognition of shellable complexes [GPP ${ }^{+}$19]. Though the hardness reduction requires the implication only in dimension 2 , we find it interesting to provide a higher-dimensional generalization. For example, the computational complexity status of recognition of shellable/collapsible 3 -spheres is unknown and the implication (iii) $\Rightarrow$ (ii) could provide a link between the two notions.

For explaining our generalization, we briefly introduce some notions (see also Section 2 for the notions undefined here).

Collapsibility. Let $\mathbf{K}$ be a simplicial complex and $\sigma \in \mathbf{K}$ be a face which is contained in only one face $\tau \in K$ with $\sigma \subsetneq \tau$. (Necessarily $\operatorname{dim} \tau=\operatorname{dim} \sigma+1$ and $\tau$ is a facet $\mathbf{K}$, that is, an inclusion-wise maximal face of $\mathbf{K}$ ). In this case, we say that $\sigma$ is a free face of $\mathbf{K}$ and we also say that a complex $\mathbf{K}^{\prime}$ arises from $\mathbf{K}$ by an elementary collapse if there are $\sigma$ and $\tau$ as above such that $\mathbf{K}^{\prime}=\mathbf{K} \backslash\{\sigma, \tau\}$, we denote this by $\mathbf{K} \searrow \mathbf{K}^{\prime}$. A complex $\mathbf{K}$ is collapsible, if there is a sequence $\left(\mathbf{K}_{1}, \ldots, \mathbf{K}_{r}\right)$ of complexes such that $\mathbf{K}_{1}=\mathbf{K}, \mathbf{K}_{r}$ is a point, and $\mathbf{K}_{1} \searrow \mathbf{K}_{2} \searrow \cdots \searrow \mathbf{K}_{r}$. An important property of collapsibility is that the elementary collapses preserve the homotopy type, a fortiori, the homology groups.

Shellability. Let $\mathbf{K}$ be a simplicial complex of dimension $k$. A total order $F_{1}, \ldots, F_{t}$ of facets of $\mathbf{K}$ is called a shelling if $F_{i} \cap \bigcup_{j=1}^{i-1} F_{j}$ is a pure $(k-1)$-dimensional complex. (Purely formally, we consider the facets in the formula $F_{i} \cap \bigcup_{j=1}^{i-1} F_{j}$ above as subcomplexes of $\mathbf{K}$.) $\mathbf{K}$ is then said to be shellable if it admits a shelling order. For comparison with collapsibility, we will also use the reverse shelling order $F_{t}, \ldots, F_{1}$.

Removal-collapsibility condition. We will say that a pure complex $\mathbf{K}$ satisfies the removalcollapsibility condition, abbreviated to (RC) condition, if $\mathbf{K}$ is either empty or $\mathbf{K}$ becomes collapsible after removing some number of facets. We remark that if $\operatorname{dim} \mathbf{K}=d$ the number of removed facets can be easily computed as $\tilde{\beta}_{d}\left(\mathbf{K} ; \mathbb{Z}_{2}\right)$ where $\tilde{\beta}_{d}\left(\mathbf{K} ; \mathbb{Z}_{2}\right)$ denotes the reduced $d$ th Betti number, i.e., the rank of the reduced homology group $\tilde{H}_{d}\left(\mathbf{K} ; \mathbb{Z}_{2}\right) 1_{1}^{1}$ Indeed, by a routine application of the Mayer-Vietoris exact sequence, removing a facet either decreases $\tilde{\beta}_{d}\left(\mathbf{K} ; \mathbb{Z}_{2}\right)$ by one or increases $\tilde{\beta}_{d-1}\left(\mathbf{K} ; \mathbb{Z}_{2}\right)$ by one. But we cannot afford the latter case if the complex becomes collapsible after removing some number of facets. In addition, the lower dimensional homology remains unaffected when removing a facet (directly from the definition of simplicial homology or again by a Mayer-Vietoris exact sequence), therefore a complex satisfying (RC) condition also satisfies $\tilde{\beta}_{i}\left(\mathbf{K} ; \mathbb{Z}_{2}\right)=0$ for $0 \leq i \leq d-1$. In particular, $\tilde{\chi}(K)=(-1)^{d} \tilde{\beta}_{d}\left(\mathbf{K} ; \mathbb{Z}_{2}\right)$.

We also observe that if $d=1$, that is, if $\mathbf{K}$ is a graph, then the (RC) condition is equivalent with stating that $\mathbf{K}$ is connected. Also, every 0-complex satisfies the (RC) condition.

Altogether, Hachimori's condition (iii) for 2-complexes is equivalent to saying that the link of the empty face (i. e., K) and the link of every vertex satisfies the (RC) condition. This is furthermore equivalent with saying that the link of every face of $\mathbf{K}$ satisfies the (RC) condition as links of dimension at most 0 always satisfy the $(\mathrm{RC})$ condition. We say that $\mathbf{K}$ satisfies

\footnotetext{
${ }^{1}$ The choice of coefficients $\mathbb{Z}_{2}$ is not very important here. We could choose an arbitrary field.
} 
the hereditary removal-collapsibility condition, abbreviated to (HRC) condition, if the link of every face of $\mathbf{K}$ satisfies the (RC) condition. In particular, (HRC) is equivalent to Hachimori's condition (iii) for 2-complexes. This condition is hereditary in the following sense: If $\mathbf{K}$ satisfies (HRC) and $\sigma \in \mathbf{K}$, then the link $\operatorname{lk}(\sigma, \mathbf{K})$ also satisfies (HRC). Indeed, the link of $\sigma^{\prime}$ in $\operatorname{lk}(\sigma, \mathbf{K})$ is just the link of $\sigma \cup \sigma^{\prime}$ in $\mathbf{K}{ }^{2}$

We establish the following generalization of Hachimori's implication (iii) $\Rightarrow$ (ii).

Theorem 1. Let $\mathbf{K}$ be a pure simplicial d-complex satisfying the (HRC) condition, then the second barycentric subdivision $\mathrm{sd}^{2} \mathbf{K}$ is shellable.

We suspect that the reverse implication does not hold but we are not aware of a concrete complex violating the reverse implication. Possibly interesting examples could be the non-collapsible triangulations of the 3-ball $B_{15,66}$ and $B_{17,95}$ constructed by Benedetti and Lutz [BL13] but we do not know if their second barycentric subdivisions are shellable.

For the proof of Theorem 1, we will define two coarser notions than shellability called star decomposability and star decomposability in vertices, which may be of independent interest. Together with vertex decomposability of Provan and Billera [PB80] we will establish the following chain of implications, where the last implication is a result of Provan and Billera.

star decomposable in vertices $\Rightarrow$ star decomposable $\Rightarrow$ vertex decomposable $\Rightarrow$ shellable

Therefore, for a proof of Theorem 1 it is sufficient to prove the following generalization (together with the first two promised implications).

Theorem 2. Let $\mathbf{K}$ be a pure simplicial d-complex satisfying the (HRC) condition, then the second barycentric subdivision $\mathrm{sd}^{2} \mathbf{K}$ is star decomposable in vertices.

Additional motivation and background. Both notions, collapsibility and shellability, play an important role in PL topology because they may help to determine not only the homotopy type of a given collapsible/shellable space but sometimes even the (PL) homeomorphism type. For example, a collapsible PL manifold is a ball, and a shelling of a PL-manifold (if it does not change the homotopy type) preserves the homeomorphism type RS82].

A relation between collapsibility or shellability of some subdivision of a complex and of some barycentric subdivision has been studied by Adiprasito and Benedetti [AB17]. Namely, they show that a simplicial complex is PL homeomorphic to a shellable complex if and only if it is shellable after finitely many barycentric subdivisions ${ }^{3}$ and they show an analogous result for collapsibility. If we were interested only in shellability of some barycentric subdivision of $\mathbf{K}$ in Theorem 1, it is possible that the proof could be easier, because it would be possible to use arbitrary suitable subdivisions in the intermediate steps.

Hachimori's implication (iii) $\Rightarrow$ (ii), as well as its generalization, Theorem 1, can be understood as a tool for showing that a concrete complex is shellable. A lot of effort has been devoted to developing such tools in various contexts; see e.g. [BW83, Koz97]. The advantage of Theorem 1 could be that the (HRC) condition may naturally follow from the topologi$\mathrm{cal} /$ combinatorial properties of a considered problem as it is in the case of the application of Hachimori's result in [GPP $\left.{ }^{+} 19\right]$. A possible disadvantage could be that we have to allow some flexibility on the target complex (it has to be the second barycentric subdivision of another complex).

An additional piece of motivation may come from commutative algebra. For example, Herzog and Takayama [HT02 found out that if $\mathbf{K}$ is a complex (not necessarily pure) and $I_{\mathbf{K}}$ is the

\footnotetext{
${ }^{2}$ Note that we do not claim that (HRC) is hereditary with respect to subcomplexes or induced subcomplexes.

${ }^{3}$ The result is stated in terms of derived subdivisions but there is no difference on the combinatorial level.
} 


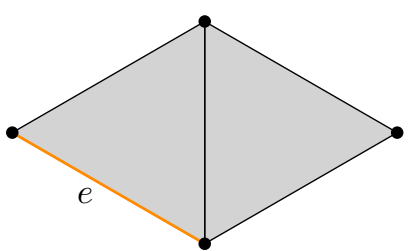

K

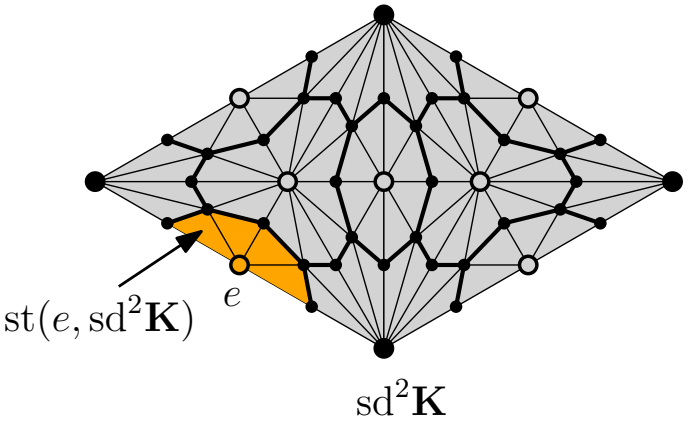

Figure 1: Decomposition of $\operatorname{sd}^{2} \mathbf{K}$ into stars. For example, an edge $e$ of $\mathbf{K}$ becomes a vertex in $\mathrm{sd}^{2} \mathbf{K}$. Consequently, its star in $\mathrm{sd}^{2} \mathbf{K}$ is one of the stars in the decomposition.

Stanley-Reisner ideal corresponding to $\mathbf{K}$, then $I_{\mathbf{K}}$ has linear quotients if and only the Alexander dual $\mathbf{K}^{*}$ is shellable (in the non-pure sense, but the pure case is a special case, of course). Thus, Theorem 1 may serve as a tool showing that certain Stanley-Reisner ideals have linear quotients.

Finally, the notions of star decomposability and star decomposability in vertices that we introduce along the way may be of independent interest as inductive tools similar to collapsibility, shellability, vertex-decomposability, etc. Although their definitions are slightly technical, they appear very naturally in our context, as we sketch in the proof strategy below. It would also be interesting to know whether these notions admit some counterpart in terms of commutative algebra (similarly to the Herzog-Takayama equivalence above).

Proof strategy. Here we first sketch Hachimori's proof (iii) $\Rightarrow$ (ii), in our words though. Then we sketch the necessary steps for upgrading the proof to higher dimensions.

Let $\mathbf{K}$ be a pure 2-complex satisfying the conditions of (iii). We want to sketch a strategy how to shell $\mathrm{sd}^{2} \mathbf{K}$. For simplicity of pictures, we will assume that $\mathbf{K}$ is already collapsible (as we want to avoid the non-trivial second homology in the pictures).

The second barycentric subdivision $\mathrm{sd}^{2} \mathbf{K}$ is covered by stars of vertices of $\mathrm{sd}^{2} \mathbf{K}$ which correspond to original faces of $\mathbf{K}$; see Figure 1. The stars may overlap, but they overlap only in their boundaries (in links). Now, let us consider an elementary collapse $\mathbf{K} \searrow \mathbf{K}^{\prime}$ while removing a free face $\sigma$ and a maximal face $\tau$ containing $\sigma$. Naturally, in $\operatorname{sd}^{2} \mathbf{K}$ we want to emulate this by a reverse shelling removing the triangles first in $\operatorname{st}\left(\sigma, \mathrm{sd}^{2} \mathbf{K}\right)$ and then in $\operatorname{st}\left(\tau, \mathrm{sd}^{2} \mathbf{K}\right)\left\{\begin{array}{l}4 \\ \text { see }\end{array}\right.$ Figure 2. This is indeed a good strategy as Hachimori Hac08 showed. However, this quite heavily depends on the fact that the dimension of the complex is 2 as the structure $\operatorname{of~}^{2} \mathbf{K}$ is so simple that all steps are obvious.

In general dimension we want to proceed similarly. However it seems out of reach to describe directly the order of removals of facets of $\mathrm{sd}^{2} \mathbf{K}$ and check that this is a shelling order due to a complicated structure of $\mathrm{sd}^{2} \mathbf{K}$. At least we initially tried this approach but we quickly got lost in addressing too many cases. Therefore, we instead use the aid of some coarser notions.

The first helpful notion is vertex decomposability of Provan and Billera [PB80]. A simplicial $d$-complex $\mathbf{K}$ is vertex decomposable if it is pure and

- $\mathbf{K}$ is a $d$-simplex, or

- there is a vertex $v \in V(\mathbf{K})$ such that $\mathbf{K}-v$ is $d$-dimensional vertex decomposable (where $\mathbf{K}-v$ denotes the complex obtained by removing $v$ and all faces containing $v$ from $\mathbf{K}$ )

\footnotetext{
${ }^{4}$ Formally speaking, st $\left(\sigma, \operatorname{sd}^{2} \mathbf{K}\right)$ stands for $\operatorname{st}\left(\{\{\sigma\}\}, \operatorname{sd}^{2} \mathbf{K}\right)$, etc.; see our convention in the preliminaries.
} 


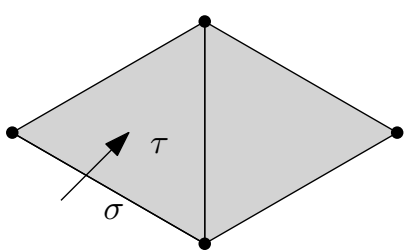

K

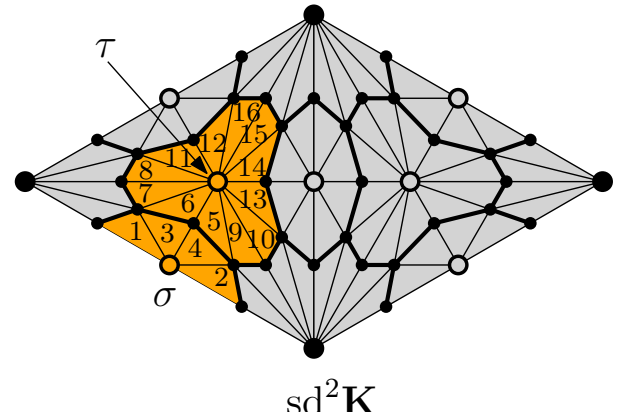

$\operatorname{sd}^{2} \mathbf{K}$

Figure 2: Reverse shelling of $\operatorname{sd}^{2} \mathbf{K}$ following an elementary collapse of $\mathbf{K}$. The numbers in triangles indicate a valid order of removing triangles.

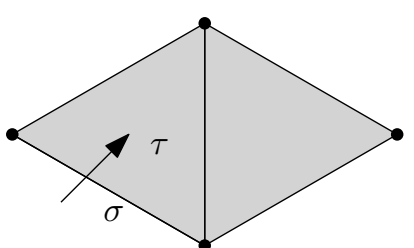

$\mathbf{K}$

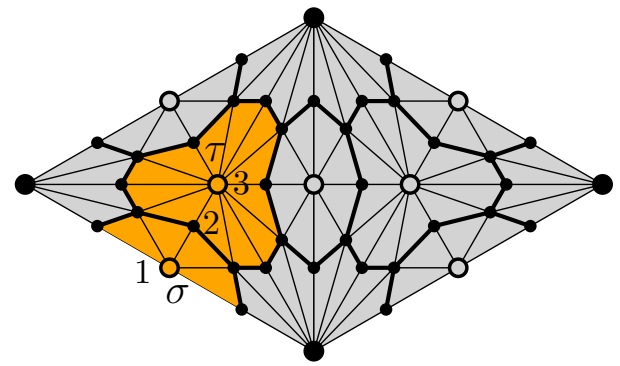

$\operatorname{sd}^{2} \mathbf{K}$

Figure 3: Vertex decomposition (shedding) of $\operatorname{sd}^{2} \mathbf{K}$ following an elementary collapse of $\mathbf{K}$. In this case, we first remove $\sigma$, then the vertex in between of $\sigma$ and $\tau$ and finally $\tau$.

and $\operatorname{lk}(v, \mathbf{K})$ is $(d-1)$-dimensional vertex decomposable.

This recursive definition induces an order $v_{1}, \ldots, v_{n-(d+1)}$ of $n-(d+1)$ vertices of $\mathbf{K}$ according to the sequence of vertex removals in the second item (where $n$ is the number of vertices of $\mathbf{K})$. This order is called a shedding order and we artificially extend any shedding order to all vertices of $\mathbf{K}$ so that the remaining vertices follow in arbitrary order. (Intuitively, as soon as we reach a $d$-simplex in the first item, we allow removing vertices in arbitrary order.)

Proving that $\mathrm{sd}^{2} \mathbf{K}$ is vertex decomposable is stronger than showing that $\mathrm{sd}^{2} \mathbf{K}$ is shellable, and it also seems easier to specify the shedding order as we deal with a smaller number of objects. For example, in case of the collapse from Figure 2, we specify the order only on three vertices; see Figure 3.

On the other hand, it is even easier to start removing the closed stars of vertices (and then taking a closure to get again a simplicial complex). In case of Figure 3, we would first remove the closed star of $\sigma$ in $\mathrm{sd}^{2} \mathbf{K}$. Subsequently, when taking the closure, we reintroduce the full link of $\sigma$. Thus in this case, our first step coincides with removing $\sigma$ (and therefore the open star of $\sigma$ ). The second step is, however, more interesting (see Figure 4): First we remove the closed star of $\tau$. Then, when taking the closure, we do not reintroduce the vertex in between of $\sigma$ and $\tau$. Therefore, this second step removes simultaneously two vertices.

This will be our notion of star decomposability; however, one of the key steps in our approach is to identify an appropriate property of order of removals as above, which implies vertex decomposability of our complex. For sketching the idea, let us again consider the case of removing the closed star of $\tau$ in the second step above. Similarly as in the case of vertex 


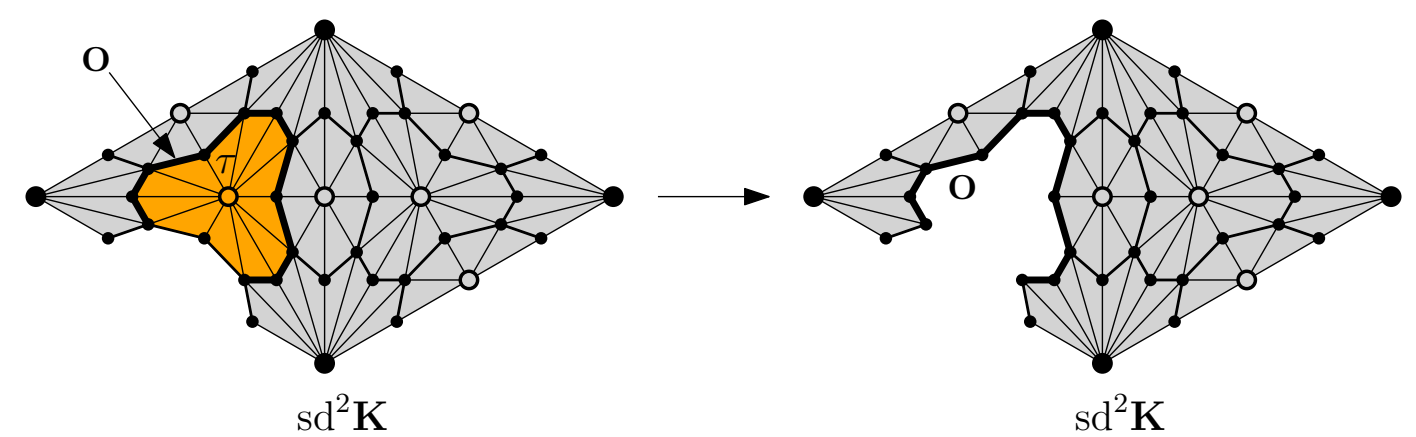

Figure 4: Overlap of the link of $\tau$ and the rest of the complex.

decomposability, we will need that the link of the center of the removed star (in this case the link of $\tau$ ) is star decomposable. However, this is not the only condition that we require. Let $\mathbf{O}$ be the overlap of the link of $\tau$ and the remainder of the complex after removing the star of $\tau$ (see Figure 4). We will actually need a star decomposition of the link of $\tau$ such that $\mathbf{O}$ is an intermediate step in this decomposition. Overall, this additional condition ensures a well working induction for deducing vertex decomposability. We postpone the precise definition of star decomposability to Section 3 .

Finally, we will utilize the fact that we are interested in star decomposability of the complex $\operatorname{sd}^{2} \mathbf{K}$ which is a barycentric subdivision of another complex, namely $\operatorname{sd}^{2} \mathbf{K}=\operatorname{sd} \mathbf{L}$ where $\mathbf{L}=$ sd $\mathbf{K}$. We will introduce the notion of star decomposability in vertices which will mean that we are removing only stars centered in vertices of $s d \mathbf{L}$ which are simultaneously vertices of $\mathbf{L}$ as in Figure 4. (Note that vertices of $\mathbf{L}$ are faces of $\mathbf{K}$.) This brings one more advantage. We will essentially need claims of the following spirit: If $\operatorname{sd}(\mathbf{X})$ and $\operatorname{sd}(\mathbf{Y})$ are star decomposable in vertices, then $\operatorname{sd}(\mathbf{X} * \mathbf{Y})$ is star decomposable in vertices as well (here $\mathbf{X} * \mathbf{Y}$ denotes the join of $\mathbf{X}$ and $\mathbf{Y}$ ). In addition, we will also need to describe the order of the star decomposition in vertices of $\operatorname{sd}(\mathbf{X} * \mathbf{Y})$. Though it is probable that analogous claims are valid also for star decomposability, vertex decomposability and/or shellability, the notion of star decomposability in vertices removes at least one layer of complications in the proof: It is just sufficient to describe the order of the decomposition of $\operatorname{sd}(\mathbf{X} * \mathbf{Y})$ as some total order on $V(\mathbf{X} * \mathbf{Y})=V(\mathbf{X}) \sqcup V(\mathbf{Y})$ via a suitable way of interlacing the total orders on $V(\mathbf{X})$ and $V(\mathbf{Y})$ (here $V(\mathbf{X}) \sqcup V(\mathbf{Y})$ denotes the disjoint union of $V(\mathbf{X})$ and $V(\mathbf{Y})$ ).

\section{Preliminaries}

In this section, we briefly overview the standard terminology regarding simplicial complexes, including some of the notions mentioned in the introduction without the definition. We generally assume that the reader is familiar with simplicial complexes. Thus the main purpose is to set up the notation.

We work with finite abstract simplicial complexes, that is, finite set systems $\mathbf{K}$ such that if $\sigma \in \mathbf{K}$ and $\sigma^{\prime} \subseteq \sigma$, then $\sigma^{\prime} \in \mathbf{K}$. Elements of $\mathbf{K}$ are faces; a $k$-face is a face of dimension $k$, that is, a face of size $k+1$. Vertices correspond to 0 -faces of $\mathbf{K}$ (specifically, a vertex $v$ corresponds to a 0 -face $\{v\}$ ); and the set of vertices is denoted $V(\mathbf{K})$. The dimension of $\mathbf{K}$ is the dimension of the largest face (or $-\infty$, if $\mathbf{K}$ is empty). The complex $\mathbf{K}$ is pure if all inclusion-wise maximal faces have the same dimension.

A join of two simplicial complexes $\mathbf{K}_{1}$ and $\mathbf{K}_{2}$ is the complex $\mathbf{K}_{1} * \mathbf{K}_{2}:=\left\{\sigma_{1} \sqcup \sigma_{2}: \sigma_{1} \in\right.$ 

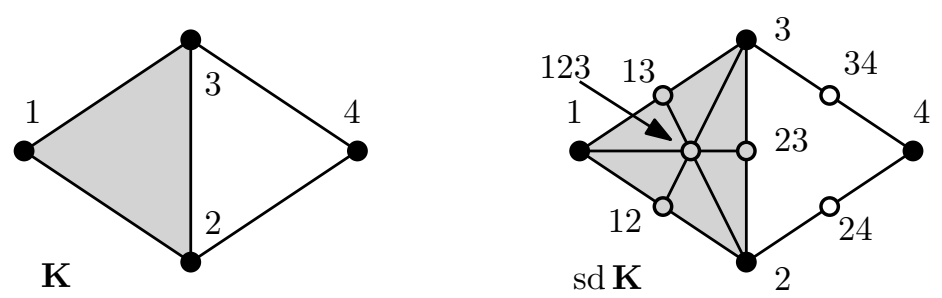

Figure 5: The barycentric subdivision sd $\mathbf{K}$ of a complex $\mathbf{K}$. The notation on the right picture is simplified so that 12 stands for $\{1,2\}$, etc.

$\left.\mathbf{K}_{1}, \sigma_{2} \in \mathbf{K}_{2}\right\}$ where $\sqcup$ stands for disjoint union ${ }^{5}$ In our inductive arguments, we will carefully distinguish the empty complex $\emptyset$ and the complex $\{\emptyset\}$ containing a single face, which is $\emptyset$. Note that $\mathbf{K} * \emptyset=\emptyset$, whereas $\mathbf{K} *\{\emptyset\}=\mathbf{K}$.

Given a face $\sigma$ of $\mathbf{K}$, the link of $\sigma$ in $\mathbf{K}$ is defined as $\operatorname{lk}(\sigma, \mathbf{K}):=\left\{\sigma^{\prime} \backslash \sigma: \sigma^{\prime} \in \mathbf{K}, \sigma \subseteq \sigma^{\prime}\right\}$. The (closed) star of $\sigma$ in $\mathbf{K}$ is defined as $\operatorname{st}(\sigma, \mathbf{K}):=\left\{\sigma^{\prime} \in \mathbf{K}: \sigma^{\prime} \cup \sigma \in \mathbf{K}\right\}$.

The barycentric subdivision of a simplicial complex $\mathbf{K}$ is the simplicial complex

$$
\operatorname{sd} \mathbf{K}:=\left\{\left\{\sigma_{1}, \ldots, \sigma_{n}\right\}: \sigma_{1}, \ldots, \sigma_{n} \in \mathbf{K}, \emptyset \neq \sigma_{1} \subsetneq \sigma_{2} \subsetneq \cdots \subsetneq \sigma_{n}\right\} .
$$

The geometric idea behind the definition of barycentric subdivision is the following: According to the definition, the vertices of sd $\mathbf{K}$ are nonempty faces of $\mathbf{K}$. Place a vertex of sd $\mathbf{K}$ into the barycenter of the face it represents in $\mathbf{K}$ (in the geometric realization of $\mathbf{K}$, which we did not define here). Then sd $\mathbf{K}$ represents a (geometric) subdivision of $\mathbf{K}$; see Figure 5. (In the subsequent text, we will not need any details about geometric realization of the barycentric subdivision. However, we will use this geometric interpretation in motivating pictures.)

Note also that if $v$ is a vertex of $\mathbf{K}$, then $\{v\}$ is a vertex of $\operatorname{sd} \mathbf{K}$. If there is no risk of confusion, we write $v$ instead of $\{v\}$ in formulas such as $1 \mathrm{k}(v, \mathrm{sd} \mathbf{K})$. We apply similar conventions to the second barycentric subdivision, so we write $\mathrm{lk}\left(v, \mathrm{sd}^{2} \mathbf{K}\right)$ instead of the cumbersome $\operatorname{lk}\left(\{\{v\}\}, \operatorname{sd}^{2} \mathbf{K}\right)$, or $\operatorname{lk}\left(\sigma, \operatorname{sd}^{2} \mathbf{K}\right)$ instead of $\operatorname{lk}\left(\{\sigma\}, \operatorname{sd}^{2} \mathbf{K}\right)$ if $\sigma$ is a face of $\mathbf{K}$.

\section{Star decomposability}

Given a simplicial complex $\mathbf{X}$ and a set $W \subseteq V(\mathbf{X})$, we say that $W$ induces a star partition of $\mathbf{X}$ if

(i) $\mathbf{X}=\bigcup_{w \in W} \operatorname{st}(w, \mathbf{X})$, and

(ii) any two distinct vertices $w_{1}, w_{2} \in W$ are not neighbors in $\mathbf{X}$.

An example of a set inducing a star partition is the set $\left\{w_{1}, w_{2}, w_{3}, w_{4}\right\}$ in Figure 6 .

Now, let us assume that $W$ induces a star partition. Given a total order $\prec$ on $W, W^{\prime} \subseteq W$, and $w \in W$, we set $W_{\succ w}^{\prime}:=\left\{w^{\prime} \in W^{\prime}: w^{\prime} \succ w\right\}$ and $W_{\succ w}^{\prime}:=\left\{w^{\prime} \in W^{\prime}: w^{\prime} \succeq w\right\}$. We will also use the notation

$$
\operatorname{st}\left(W^{\prime}, \mathbf{X}\right):=\bigcup_{w^{\prime} \in W^{\prime}} \operatorname{st}\left(w^{\prime}, \mathbf{X}\right)
$$

\footnotetext{
${ }^{5}$ We can perform the disjoint union of two sets $A$ and $B$ even if $A$ and $B$ are not disjoint. The standard model in such case is to take $A \times\{1\} \cup B \times\{2\}$.
} 


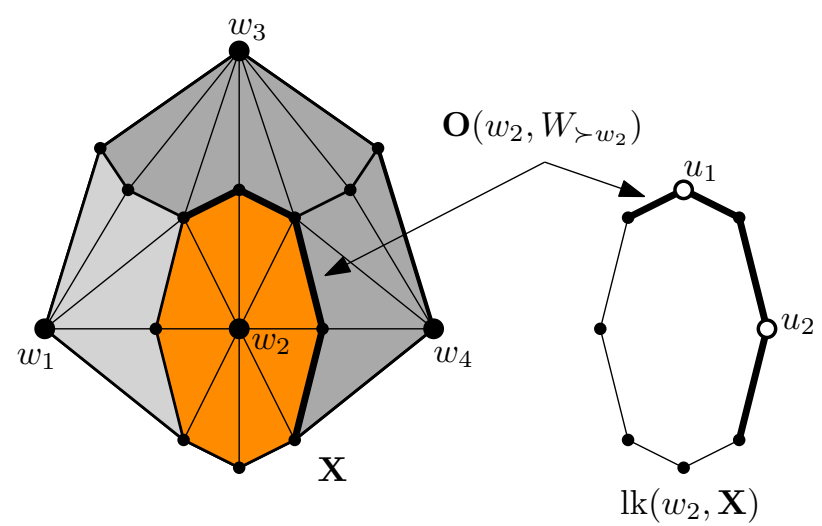

Figure 6: An example of the star decomposition induced by the set $W=\left\{w_{1}, w_{2}, w_{3}, w_{4}\right\}$ with the order $w_{1} \prec w_{2} \prec w_{3} \prec w_{4}$ (left) and an example of the set $U\left(w_{2}\right)=\left\{u_{1}, u_{2}\right\}$ such that $\operatorname{st}\left(U\left(w_{2}\right), \operatorname{lk}(w, \mathbf{X})\right)=\mathbf{O}\left(w_{2}, W_{\succ w_{2}}\right)$ and the pair $\left(\operatorname{lk}\left(w_{2}, \mathbf{X}\right), U\left(w_{2}\right)\right)$ is star decomposable (right).

for an arbitrary subset $W^{\prime}$ of $V(\mathbf{X})$. Furthermore given $x \in W$ and a set $W^{\prime} \subseteq W$, we define

$$
\mathbf{O}\left(x, W^{\prime}\right):=\operatorname{lk}(x, \mathbf{X}) \cap \operatorname{st}\left(W^{\prime}, \mathbf{X}\right)=\operatorname{lk}(x, \mathbf{X}) \cap \bigcup_{w^{\prime} \in W^{\prime}} \operatorname{st}\left(w^{\prime}, \mathbf{X}\right)=\operatorname{lk}(x, \mathbf{X}) \cap \bigcup_{w^{\prime} \in W^{\prime}} \operatorname{lk}\left(w^{\prime}, \mathbf{X}\right) .
$$

See Figure 6. Note that this is the overlap mentioned in the introduction. Occasionally, if we need to emphasize dependency on $\mathbf{X}$, we write $\mathbf{O}_{\mathbf{X}}\left(x, W^{\prime}\right)$.

Now, we are ready to introduce star decomposability. Following the sketch in the introduction, we want to introduce star decomposability of a simplicial complex $\mathbf{X}$. However, in order to formulate all conditions correctly, we need to state this definition for pairs.

Definition 3 (Star decomposability). Let $(\mathbf{X}, X)$ be a pair where $\mathbf{X}$ is a simplicial complex which is pure and $k$-dimensional, $k \geq-1$ (that is, $\mathbf{X} \neq \emptyset$ ), and $X \subseteq V(\mathbf{X})$. We inductively define star decomposability of the pair $(\mathbf{X}, X)$. We also say that $\mathbf{X}$ is star decomposable if there is $X \subseteq V(\mathbf{X})$ for which the pair $(\mathbf{X}, X)$ is star decomposable.

For $k=-1$, the pair $(\{\emptyset\}, \emptyset)$ is star decomposable.

If $k \geq 0$, then $(\mathbf{X}, X)$ is star decomposable, if there is a set $W \neq \emptyset$ inducing a star partition and a total order $\prec$ on $W$ with the following properties.

Order condition: $X=W_{\succeq w^{\prime}}$ for some $w^{\prime} \in W$.

Link condition: For any vertex $w \in W$ except for the last vertex in the order $\prec$, there is a nonempty set $U=U(w) \subseteq V(\operatorname{lk}(w, \mathbf{X}))$ such that $\operatorname{st}(U, \operatorname{lk}(w, \mathbf{X}))=\mathbf{O}\left(w, W_{\succ w}\right)$ and the pair $(\operatorname{lk}(w, \mathbf{X}), U)$ is star decomposable.

Last vertex condition: For the last vertex $\hat{w} \in W$ in the order $\prec$, the $\operatorname{link} \operatorname{lk}(\hat{w}, \mathbf{X})$ is star decomposable.

If the order $\prec$ on $W$ satisfies the three conditions above, we say that $\prec$ induces a star decomposition of $(\mathbf{X}, X)$.

See Figure 6 for an example.

\footnotetext{
${ }^{6}$ The symbol $\mathbf{O}$ in the notation stands for the 'overlap' of $\operatorname{lk}(x, \mathbf{X})$ and $\operatorname{st}\left(W^{\prime}, \mathbf{X}\right)$.
} 
Remarks 4 .

(i) Observe that the order condition implies $X \neq \emptyset$ if $k \geq 0$.

(ii) In the definition above, we remark that if $\mathbf{X}$ is $k$-dimensional and pure, for $k \geq 0$, then for any $w \in V(\mathbf{X})$, the link $\operatorname{lk}(w, \mathbf{X})$ is $(k-1)$-dimensional and pure. Therefore, in the last two conditions, we indeed refer to star decomposability of a pure complex of smaller dimension.

In addition, for any $W^{\prime} \subseteq V(\mathbf{X}), W^{\prime} \neq \emptyset$, st $\left(W^{\prime}, \mathbf{X}\right)$ is $k$-dimensional and pure. In particular, when replacing $\mathbf{X}$ with $\operatorname{lk}(w, \mathbf{X})$, we get that $\mathbf{O}\left(w, W_{\succ w}\right)=\operatorname{st}(U, \operatorname{lk}(w, \mathbf{X}))$ is $(k-1)$-dimensional and pure.

(iii) If $k=0$, then every pair $(\mathbf{X}, X)$ is star decomposable if and only if $X \neq \emptyset$. Indeed, the only if part follows from (i). For the 'if' part, we observe that we can set $W=V(\mathbf{X})$ and we can use any order $\prec$ on $W$ such that $X=W_{\succeq w^{\prime}}$ for some $w^{\prime}$. Both the link condition and the last vertex condition refer to star decomposability of $(\{\emptyset\}, \emptyset)$, which we assume.

(iv) If $k=1$, then it is not difficult to show that $\mathbf{X}$ is star decomposable if and only if $\mathbf{X}$ is a connected bipartite graph. Note that requiring that $\mathbf{X}$ is connected is a must as we want to get that star decomposability implies vertex decomposability. Here is the place where the possibly slightly mysterious property ' $X \neq \emptyset$ if $k \geq 0$ ' comes into the play. Indeed, this property and the link condition achieve that the overlap $\mathbf{O}\left(w, W_{\prec w}\right)$ is nonempty, thus $\mathbf{X}$ must be a connected graph.

\section{Star decomposability implies vertex decomposability.}

In this section, we want to describe how star decomposability implies vertex decomposability. We start with a simple (folklore) lemma verifying that some order is a shedding order (with respect to our convention that we extend the shedding order also to the vertices of the last simplex). Given a simplicial complex $\mathbf{X}$, a total (or partial) order $\prec$ on $V(\mathbf{X})$, and $v \in V(\mathbf{X})$, by $\mathbf{X}_{\succ v}$ we denote the subcomplex of $\mathbf{X}$ induced by vertices that are greater than $v$. Similarly $\mathbf{X}_{\succeq v}$ is induced by $v$ and the vertices that are greater than $v$.

Lemma 5. Let $\mathbf{X}$ be a pure $k$-dimensional simplicial complex, $k \geq 0$. Let $\prec$ be a total order on $V(\mathbf{X})$. Then $\prec i s$ a shedding order if and only if for every vertex $v$ except for the last $k+1$ vertices, the link $\operatorname{lk}\left(v, \mathbf{X}_{\succeq v}\right)$ is vertex decomposable and $(k-1)$-dimensional, and $\mathbf{X}_{\succ v}$ is pure $k$-dimensional.

Proof. The 'only if' part of the statement follows immediately from the definition of vertex decomposability and the shedding order, thus we focus on the 'if' part.

If $\mathbf{X}$ has $k+1$ vertices, then $\mathbf{X}$ is a $k$-simplex and we are done. Otherwise, we proceed by induction on the number of vertices of $\mathbf{K}$.

Let $v_{1}$ be the first vertex in the order $\prec$. Then we need to check that $\operatorname{lk}\left(v_{1}, \mathbf{X}_{\succeq v_{1}}\right)$ is vertex decomposable and $(k-1)$-dimensional, which is part of the assumptions. We also need to check that $\mathbf{X}-v_{1}=\mathbf{X}_{\succ v_{1}}$ is vertex decomposable and $k$-dimensional. Again, $k$-dimensional is part of the assumptions, thus, it remains to check that $\mathbf{X}-v_{1}$ is vertex decomposable. However, this follows from the induction applied to $\mathbf{X}_{\succ v_{1}}$ and $\prec$ restricted to $V(\mathbf{X}) \backslash\left\{v_{1}\right\}$.

Now, let $\mathbf{X}$ be a star decomposable simplicial complex, let $W$ be a subset of $V(\mathbf{X})$ which induces a star partition of $\mathbf{X}$ and let $\prec$ be a total order which induces a star decomposition 


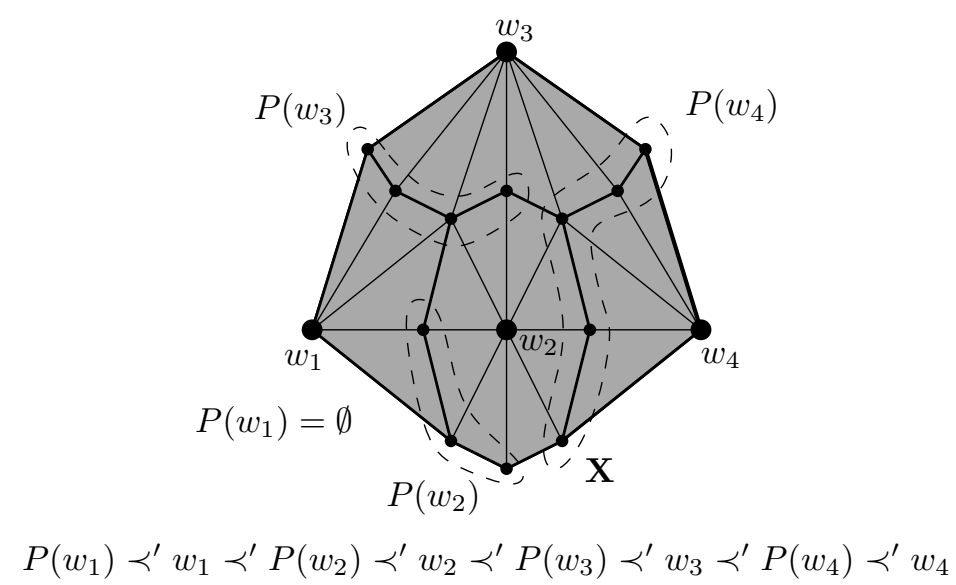

Figure 7: The set $P(w)$ and the auxiliary order $\prec^{\prime}$ for the star decomposition in Figure 6 .

of $\mathbf{X}$. We will define a suitable partial order $\prec^{\prime}$ on $V(\mathbf{X})$ extending $\prec$ such that the desired shedding order in the vertex decomposition of $\mathbf{X}$ will follow $\prec$ '.

For arbitrary $v \in V(\mathbf{X})$, let $p(v)$ be the last vertex in the $\prec$ order among the vertices $w \in W$ such that $v \in \operatorname{st}(w, \mathbf{X})$. In particular $p(w)=w$ for any $w \in W$. If we want to emphasize $\prec$, we write $p(v, \prec)$ (which will be used in a single but important case of the proof of Theorem 7). Now, we define $\prec^{\prime}$ in the following way. We set $v \prec^{\prime} v^{\prime}$ if $p(v) \prec p\left(v^{\prime}\right)$ for $v, v^{\prime} \in V(\mathbf{X})$. In addition, we set $v \prec^{\prime} w$ if $p(v)=w$ and $v \neq w$. Finally, if $p(v)=p\left(v^{\prime}\right)$ and $v, v^{\prime} \notin W$, then $v$ and $v^{\prime}$ are incomparable in $\prec^{\prime}$. We say that $\prec^{\prime}$ is derived from $\prec$. An example of this order is given in Figure 7 where $P(w)=\{v \in V(\mathbf{X}): v \neq w, p(v)=w\}$ for $w \in W$; the elements in $P(w)$ are incomparable.

We will often need that $\operatorname{st}\left(W_{\succ w}, \mathbf{X}\right)$ is an induced subcomplex of $\mathbf{X}$ for $w \in W \backslash\{\hat{w}\}$ :

Lemma 6. Let $\mathbf{X}$ be a star decomposable complex, let $W$ be a subset of $V(\mathbf{X})$ which induces a star partition of $\mathbf{X}$ and let $\prec$ be a total order on $W$ which induces a star decomposition of $\mathbf{X}$. Let $\prec^{\prime}$ be the partial order on $V(\mathbf{X})$ derived from $\prec$ and let $w \in W$ be different from the last vertex $\hat{w}$. Then $\operatorname{st}\left(W_{\succ w}, \mathbf{X}\right)$ is the induced subcomplex $\mathbf{X}_{\succ^{\prime} w}$ of $\mathbf{X}$.

Proof. If $\operatorname{dim} \mathbf{X}=-1$, then the statement is void. If $\operatorname{dim} \mathbf{X}=0$, the assertion easily follows from Remark 4(iii). Thus, we may assume $\operatorname{dim} \mathbf{X} \geq 1$, which we will implicitly when referring to the link condition.

Recall that $\operatorname{st}\left(W_{\succ w}, \mathbf{X}\right)=\bigcup_{w^{+} \in W_{\succ w}} \operatorname{st}\left(w^{+}, \mathbf{X}\right)$. It is easy to check the inclusion $\mathbf{X}_{\succ^{\prime} w} \supseteq$ $\bigcup_{w^{+} \in W_{\succ w}} \operatorname{st}\left(w^{+}, \mathbf{X}\right)$ because $\operatorname{st}\left(w^{+}, \mathbf{X}\right) \subseteq \mathbf{X}_{\succ^{\prime} w}$ for every $w^{+} \succ w$. (Note that if $v$ is a neighbor of $w^{+}$in $\mathbf{X}$, then $p(v) \succeq w^{+} \succ w$. Thus, $v$ belongs to $V\left(\mathbf{X}_{\succ^{\prime} w}\right)$.) Therefore, it remains to show $\mathbf{X}_{\succ^{\prime} w} \subseteq \bigcup_{w^{+} \in W_{\succ w}} \operatorname{st}\left(w^{+}, \mathbf{X}_{\succ^{\prime} w}\right)$.

Let $\sigma \in \mathbf{X}_{\succ^{\prime} w}$. For contradiction, let us assume that $\sigma \notin \operatorname{st}\left(w^{+}, \mathbf{X}\right)$ for all $w^{+} \succ w$. (In particular, $\sigma \neq \emptyset$.) Let $w^{-} \preceq w$ be the largest vertex in $W$ (according to the total order $\prec$ ) such that $\sigma \in \operatorname{st}\left(w^{-}, \mathbf{X}\right)$. Such $w^{-}$must exist because $W$ induces a star partition of $\mathbf{X}$. In addition, because $\sigma \in \mathbf{X}_{\succ^{\prime} w}$ and $w^{-} \preceq w$, we get that $w^{-} \notin \sigma$. Thus, $\sigma \in \operatorname{lk}\left(w^{-}, \mathbf{X}\right)$.

Now, we use that $\mathbf{X}$ is star decomposable. Namely, we use the link condition for $w^{-}$. There is $U \subseteq V(\operatorname{lk}(w, \mathbf{X}))$ such that $\operatorname{st}\left(U, \operatorname{lk}\left(w^{-}, \mathbf{X}\right)\right)=\mathbf{O}\left(w^{-}, W_{\succ w^{-}}\right)$and the pair $\left(\operatorname{lk}\left(w^{-}, \mathbf{X}\right), U\right)$ is star decomposable. By the order condition for this pair, there is a set $Z \neq \emptyset$ inducing a star partition of $\operatorname{lk}\left(w^{-}, \mathbf{X}\right)$ and a total order $\triangleleft$ on $Z$ such that $U=Z_{\unrhd z^{\prime}}$ for some $z^{\prime} \in Z$. Because $\sigma \in \operatorname{lk}\left(w^{-}, \mathbf{X}\right)$ and $Z$ induces a star partition of $\operatorname{lk}\left(w^{-}, \mathbf{X}\right)$ some vertex $v$ of $\sigma$ has to belong to $Z$. If $v \in U$, then $\sigma \in \operatorname{st}\left(U, \operatorname{lk}\left(w^{-}, \mathbf{X}\right)\right)=\mathbf{O}\left(w^{-}, W_{\succ w^{-}}\right)$which contradicts the fact that $w^{-}$is 
the largest vertex such that $\sigma \in \operatorname{st}\left(w^{-}, \mathbf{X}\right)$. If $v \in Z \backslash U$, then $p(v)=w^{-}$which contradicts $\sigma \in \mathbf{X}_{\succ^{\prime} w}$.

Now, we are ready to state and prove that star decomposability implies vertex decomposability. As the reader may expect, the order $\prec^{\prime}$ appears in the statement to allow a well working induction.

Theorem 7. Let $\mathbf{X}$ be a star decomposable simplicial complex; let $W$ be a subset of $V(\mathbf{X})$ which induces a star partition of $\mathbf{X}$; and let $\prec$ be a total order which induces a star decomposition of $\mathbf{X}$. Let $\prec^{\prime}$ be the partial order on $V(\mathbf{X})$ derived from $\prec$. Then $\mathbf{X}$ is vertex decomposable in a shedding order extending $\prec$ '.

Proof. We prove the statement by induction on $k$, the dimension of $\mathbf{X}$. If $k=-1$, the complex $\{\emptyset\}$ is vertex decomposable according to the definition of vertex decomposability (it is regarded as a -1-simplex). Although it could be covered by the second induction step, we can observe that the case $k=0$ is also easy as any order of removing vertices from a 0 -complex is a shedding order.

Now, let us prove the theorem for some $k \geq 1$ assuming that it is valid for lower values.

We first describe a total order $\prec^{\prime \prime}$ on $V(\mathbf{X})$ extending $\prec^{\prime}$. Then we verify that $\prec^{\prime \prime}$ is a shedding order. Recall that for $w \in W, P(w)$ is the set of vertices $v \in V(\mathbf{X})$ such that $p(v)=w$ but $v \neq w$; see Figure 7. To describe $\prec$ " it remains to describe $\prec$ " on each $P(w)$ separately. We distinguish whether $w$ is the last vertex in $\prec$.

If $w=\hat{w}$ is the last vertex, then $P(\hat{w})=V(\operatorname{lk}(\hat{w}, \mathbf{X}))$. By the last vertex condition (for star decomposability) $\mathrm{lk}(\hat{w}, \mathbf{X})$ is star decomposable, therefore vertex decomposable by induction as well. We set $\prec$ " on $P(\hat{w})$ as an arbitrary shedding order of $\operatorname{lk}(\hat{w}, \mathbf{X})$.

If $w$ is not the last vertex, then $P(w)=V(\operatorname{lk}(w, \mathbf{X})) \backslash V\left(\mathbf{O}\left(w, W_{\succ w}\right)\right)$. By the link condition, the pair $(\operatorname{lk}(w, \mathbf{X}), U)$ is star decomposable where $U \subseteq V(\operatorname{lk}(w, \mathbf{X}))$ satisfies $\operatorname{st}(U, \operatorname{lk}(w, \mathbf{X}))=$ $\mathbf{O}\left(w, W_{\succ w}\right)$.

Claim 7.1. Let $w \in W$ be different from the last vertex $\hat{w}$. Then the link $\operatorname{lk}(w, \mathbf{X})$ is vertex decomposable in some shedding order $\triangleleft^{\prime \prime}$ that starts on $P(w)=V(\operatorname{lk}(w, \mathbf{X})) \backslash V\left(\mathbf{O}\left(w, W_{\succ w}\right)\right)$ and then continues on $V\left(\mathbf{O}\left(w, W_{\succ w}\right)\right)$.

Proof. Consider a set $Z \subseteq V(\operatorname{lk}(w, \mathbf{X}))$ inducing a star partition of $\operatorname{lk}(w, \mathbf{X})$ and a total order $\triangleleft$ on $Z$ witnessing that the pair $(\operatorname{lk}(w, \mathbf{X}), U)$ is star decomposable. In particular, $U=Z_{\triangleright z^{\prime}}$ for some $z^{\prime} \in Z$ by the order condition. Let $\triangleleft^{\prime}$ be the partial order on $V(\operatorname{lk}(w, \mathbf{X}))$ derived from $\triangleleft$. By induction, $\operatorname{lk}(w, \mathbf{X})$ is vertex decomposable in a shedding order $\triangleleft^{\prime \prime}$ extending $\triangleleft^{\prime}$.

In addition, by the link condition (on star decomposable $\mathbf{X}$ ) we get

$$
\mathbf{O}\left(w, W_{\succ w}\right)=\operatorname{st}(U, \operatorname{lk}(w, \mathbf{X}))=\operatorname{st}\left(Z_{\unrhd z^{\prime}}, \operatorname{lk}(w, \mathbf{X})\right) .
$$

The vertices of $\operatorname{st}\left(Z_{\unrhd z^{\prime}}, \operatorname{lk}(w, \mathbf{X})\right)$ are exactly the vertices of $\operatorname{lk}(w, \mathbf{X})$ with $p(v, \triangleleft) \in Z_{\unrhd z^{\prime}}$. Therefore all vertices in $V(\operatorname{lk}(w, \mathbf{X})) \backslash V\left(\mathbf{O}\left(w, W_{\succ w}\right)\right)$ precede the vertices in $V\left(\mathbf{O}\left(w, W_{\succ w}\right)\right)=$ $V\left(\operatorname{st}\left(Z_{\unrhd z^{\prime}}, \operatorname{lk}(w, \mathbf{X})\right)\right)$ in the order $\triangleleft^{\prime}$, a fortiori, in the order $\triangleleft^{\prime \prime}$, as we need.

Now, we set $\prec^{\prime \prime}$ on $P(w)$ as the shedding order $\triangleleft^{\prime \prime}$ on $\operatorname{lk}(w, \mathbf{X})$ from Claim 7.1, restricted to $P(w)$; see Figure 8

It remains to check that $\prec^{\prime \prime}$ is the required shedding order which we do via Lemma 5 . Namely, given a vertex $v \in V(\mathbf{X})$ which is not one of the last $k+1$ vertices, we need to check that $\operatorname{lk}\left(v, \mathbf{X}_{\succeq{ }^{\prime \prime} v}\right)$ is vertex decomposable and $(k-1)$-dimensional and that $\mathbf{X}_{\succ}{ }^{\prime \prime} v$ is pure $k$-dimensional. We distinguish whether $v \in W$. 


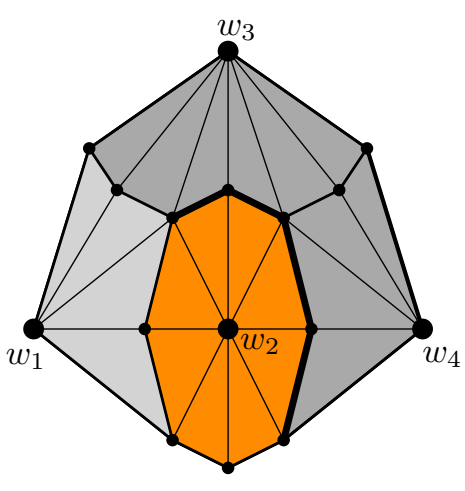

$\mathbf{X}$

$$
\begin{aligned}
& a_{1} \triangleleft a_{2} \triangleleft u_{1} \triangleleft u_{2} \\
& a_{1} \triangleleft^{\prime} b_{1} \triangleleft^{\prime} a_{2} \triangleleft^{\prime} b_{2} \triangleleft^{\prime} u_{1} \triangleleft^{\prime}\left\{b_{3}, b_{4}\right\} \triangleleft^{\prime} u_{2} \\
& w_{1} \prec^{\prime \prime} a_{1} \prec^{\prime \prime} b_{1} \prec^{\prime \prime} a_{2} \prec^{\prime \prime} w_{2} \prec^{\prime \prime} \ldots
\end{aligned}
$$

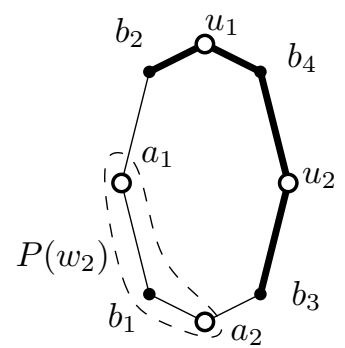

$\operatorname{lk}\left(w_{2}, \mathbf{X}\right)$

Figure 8: Setting up the order $\prec^{\prime \prime}$ on $P\left(w_{2}\right)$. The order $\triangleleft$ on $Z=\left\{a_{1}, a_{2}, u_{1}, u_{2}\right\}$ induces a star decomposition of $\left(\operatorname{lk}\left(w_{2}, \mathbf{X}\right), U\right)$ where $U=\left\{u_{1}, u_{2}\right\}$. Then $\triangleleft^{\prime}$ is the corresponding partial order on $V\left(\operatorname{lk}\left(w_{2}, \mathbf{X}\right)\right.$ ) (similarly as $\prec^{\prime}$ corresponds to $\prec$ ). Finally, we take a shedding order $\triangleleft^{\prime \prime}$ on $\operatorname{lk}\left(w_{2}, \mathbf{X}\right)$ extending $\triangleleft^{\prime}$ (by induction) and restrict it to $P\left(w_{2}\right)$ obtaining $\prec^{\prime \prime}$.

Case 1, $v \in W$ : We observe that $v$ is not the last vertex $\hat{w}$ of $\prec$ as $\hat{w}$ is also the last vertex of $\prec$ '. This allows to describe $\operatorname{lk}\left(v, \mathbf{X}_{\succeq{ }^{\prime \prime} v}\right)$ as an overlap.

Claim 7.2. $\operatorname{lk}\left(v, \mathbf{X}_{\succeq^{\prime \prime} v}\right)=\mathbf{O}\left(v, W_{\succ v}\right)$.

Proof. According to the definition of the overlap, we have $\mathbf{O}\left(v, W_{\succ v}\right)=\operatorname{lk}(v, \mathbf{X}) \cap \operatorname{st}\left(W_{\succ v}, \mathbf{X}\right)$.

First, let us assume that $\sigma \in \operatorname{lk}(v, \mathbf{X}) \cap \operatorname{st}\left(W_{\succ v}, \mathbf{X}\right)$. Each vertex $v^{\prime}$ of $\operatorname{st}\left(W_{\succ v}, \mathbf{X}\right)$ satisfies $p\left(v^{\prime}\right) \succ v$ which implies $v^{\prime} \succ^{\prime \prime} v$. Therefore, each vertex of $\sigma \cup\{v\}$ belongs to $V\left(\mathbf{X}_{\succeq^{\prime \prime} v}\right)$. Because $\sigma$ simultaneously belongs to $\operatorname{lk}(v, \mathbf{X})$, we get that it belongs to $\operatorname{lk}\left(v, \mathbf{X}_{\succeq{ }^{\prime \prime} v}\right)$.

Now, for the second inclusion, let us assume that $\sigma \in \operatorname{lk}\left(v, \mathbf{X}_{\succeq \prime \prime}\right)$. Immediately, $\sigma \in \operatorname{lk}(v, \mathbf{X})$. Because $\sigma \in \mathbf{X}_{\succ^{\prime} v}=\mathbf{X}_{\succ^{\prime \prime} v}$, Lemma 6 gives $\sigma \in \operatorname{st}\left(W_{\succ v}, \mathbf{X}\right)$.

By Claim 7.2, $\operatorname{lk}\left(v, \mathbf{X}_{\succeq \prime v v}\right)=\mathbf{O}\left(v, W_{\succ v}\right)$ which is $(k-1)$-dimensional by Remark 4(ii). In addition, $\operatorname{lk}\left(v, \mathbf{X}_{\succeq \prime}{ }^{\prime \prime}\right)$ is vertex decomposable, as we checked that $l \mathrm{k}(v, \mathbf{X})$ is vertex decomposable in some shedding order starting with $P(v)=V(\operatorname{lk}(v, \mathbf{X})) \backslash V\left(\mathbf{O}\left(v, W_{\succ v}\right)\right)$ and continuing with $V\left(\mathbf{O}\left(v, W_{\succ v}\right)\right)$; see Claim 7.1. Also $\mathbf{X}_{\succ{ }^{\prime \prime} v}=\mathbf{X}_{\succ^{\prime} v}=\operatorname{st}\left(W_{\succ v}, \mathbf{X}\right)$ by Lemma 6. Therefore $\mathbf{X}_{\succ^{\prime \prime} v}$ is pure $k$-dimensional by Remark 4 (ii). This finishes Case 1.

Case 2, $v \notin W$ : Let $w:=p(v) \in W$. Note that, in particular, $w \succ^{\prime} v$. We first check that $\operatorname{lk}\left(v, \mathbf{X}_{\succeq \prime \prime v}\right)$ is vertex decomposable and $(k-1)$-dimensional. This will follow from the following two claims.

Claim 7.3. The link $\operatorname{lk}\left(v, \mathbf{X}_{\succeq{ }^{\prime \prime} v}\right)$ is the join of $w$ and $\operatorname{lk}\left(\{v, w\}, \mathbf{X}_{\succeq^{\prime \prime} v}\right)$.

Proof. The link $\operatorname{lk}\left(\{v, w\}, \mathbf{X}_{\succeq \prime \prime v}\right)$ consists of simplices $\sigma \in \mathbf{X}_{\succeq \prime \prime}$ satisfying $v, w \notin \sigma$, and $\sigma \cup$ $\{v, w\} \in \mathbf{X}_{\succeq \prime \prime}$. Therefore, the join of $w$ and $\operatorname{lk}\left(\{v, w\}, \mathbf{X}_{\succeq{ }^{\prime \prime} v}\right)$, considered as a subcomplex of $\mathbf{X}_{\succeq \text { " } v}$, consists of simplices $\sigma \in \mathbf{X}_{\succeq \prime}$ satisfying

$$
v \notin \sigma, \text { and } \sigma \cup\{v, w\} \in \mathbf{X}_{\succeq^{\prime \prime} v} .
$$

On the other hand, $\operatorname{lk}\left(v, \mathbf{X}_{\succeq^{\prime \prime} v}\right)$ consists of simplices $\sigma \in \mathbf{X}_{\succeq \succeq^{\prime \prime} v}$ satisfying

$$
v \notin \sigma, \text { and } \sigma \cup\{v\} \in \mathbf{X}_{\succeq^{\prime \prime} v} .
$$




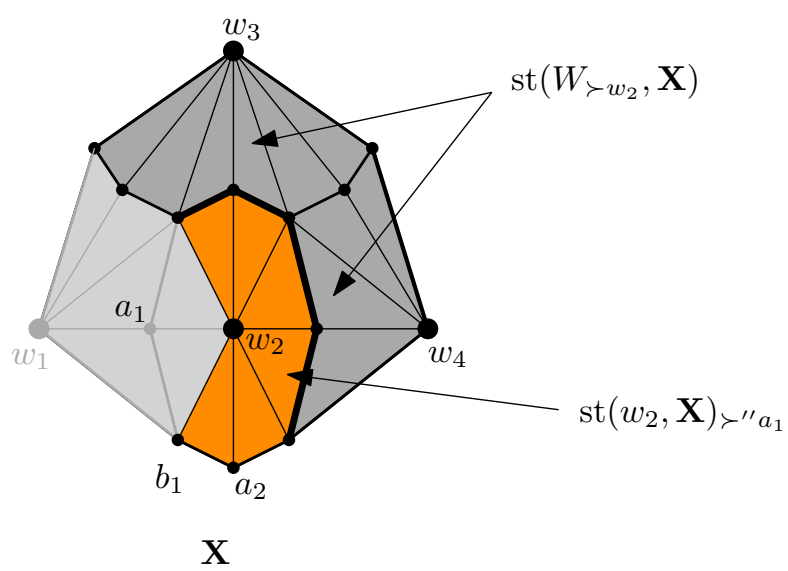

Figure 9: The complex $\mathbf{X}_{\succ}{ }^{\prime \prime} a_{1}$ as the union of $\operatorname{st}\left(W_{\succ w_{2}}, \mathbf{X}\right)$ and $\operatorname{st}\left(w_{2}, \mathbf{X}\right)_{\succ}{ }^{\prime \prime} a_{1}$. Here we use the order $\succ^{\prime \prime}$ from Figure 8 .

A simplex $\sigma \in \mathbf{X}_{\succeq{ }^{\prime \prime} v}$ satisfying (2) immediately satisfies (3) as well. Thus, it remains to consider a simplex $\sigma \in \mathbf{X}_{\succeq{ }^{\prime \prime} v}$ satisfying (3); and to show that it satisfies (2).

First, we want to deduce that $\sigma \cup\{v\}$ belongs to $\operatorname{st}\left(w^{\prime}, \mathbf{X}\right)$ for some $w^{\prime} \succeq w$. If $w$ is the first vertex of $W$ in the order $\prec$, then this claim follows from the fact that $W$ induces a star partition of $\mathbf{X}$. If $w$ is not the first vertex of $W$, let $w^{-}$be the vertex that immediately precedes $w$ in the order $\prec$. Note that $\sigma \in \mathbf{X}_{\succ^{\prime} w^{-}}$. By Lemma $6, \sigma \cup\{v\}$ belongs to $\operatorname{st}\left(w^{\prime}, \mathbf{X}\right)$ for some $w^{\prime} \succ w^{-}$, that is, $w^{\prime} \succeq w$ as required.

Now, because $p(v)=w$, the only option is that $w^{\prime}=w$. Therefore, $\sigma \cup\{v\} \in \operatorname{st}(w, \mathbf{X})$; that is, $\sigma \cup\{v, w\} \in \mathbf{X}$. Because all vertices of $\sigma \cup\{v, w\}$ belong to $\mathbf{X}_{\succeq^{\prime \prime} v}, \sigma$ satisfies (2).

Claim 7.4. The link $\operatorname{lk}\left(\{v, w\}, \mathbf{X}_{\succeq^{\prime \prime} v}\right)$ is vertex decomposable and $(k-2)$-dimensional.

Proof. We will deduce the claim from the 'only if' part of Lemma 5 used with the pure $(k-1)$ dimensional complex $\operatorname{lk}(w, \mathbf{X})$ and the shedding order $\triangleleft^{\prime \prime}$, coming from Claim 7.1. Let us recall that $\prec$ ' is defined so that it coincides with $\triangleleft^{\prime \prime}$ on $\operatorname{lk}(w, \mathbf{X})$ restricted to $P(w)$. Because $v \in P(w)$, we in particular get that $\operatorname{lk}(w, \mathbf{X})_{\succ{ }^{\prime \prime} v}=\operatorname{lk}(w, \mathbf{X})_{\unrhd^{\prime \prime} v}$.

In order to apply Lemma 5, we also check that $v$ is not among the last $k$ vertices of the aforementioned shedding $\triangleleft^{\prime \prime}$ of $\operatorname{lk}(w, \mathbf{X})$. If $w=\hat{w}$, we get this because we assume that $v$ is not among the last $k+1$ vertices in the $\prec$ " order on $V(\mathbf{X})$ (the last one is $\hat{w}$, and the vertices of $P(\hat{w})$ immediately precede). If $w \neq \hat{w}$ we get this because the overlap $\mathbf{O}\left(w, W_{\succ w}\right)$ is $(k-1)$ dimensional (see Remark $4($ ii $)$ ), and the vertices of this overlap belong to $V(\operatorname{lk}(w, \mathbf{X}))$ while they do not belong to $P(w)$.

Now, using Lemma 5 as explained above, we get that $\operatorname{lk}\left(v, \operatorname{lk}(w, \mathbf{X})_{\succeq^{\prime \prime} v}\right)=\operatorname{lk}\left(v, \operatorname{lk}(w, \mathbf{X})_{\unrhd^{\prime \prime} v}\right)$ is vertex decomposable and $(k-2)$-dimensional. Finally, $\operatorname{lk}\left(v, \operatorname{lk}(w, \mathbf{X})_{\succeq{ }^{\prime \prime} v}\right)=\operatorname{lk}\left(\{v, w\}, \mathbf{X}_{\succeq{ }^{\prime \prime} v}\right)$ because $\mathbf{X}_{\succeq \prime \prime}$ is an induced subcomplex of $\mathbf{X}$.

It follows immediately from Claims 7.3 and 7.4 that $\mathrm{lk}\left(v, \mathbf{X}_{\succeq^{\prime \prime} v}\right)$ is $(k-1)$-dimensional. In addition, because the join of two vertex decomposable complexes is vertex decomposable PB80, Proposition 2.4], we also get that $\mathrm{lk}\left(v, \mathbf{X}_{\succ \prime \prime}\right)$ is vertex decomposable.

Finally, we need to check that $\mathbf{X}_{\succ^{\prime \prime} v}$ is pure $k$-dimensional. We need one more claim; see also Figure 9 ,

Claim 7.5. If $w \neq \hat{w}$, then $\mathbf{X}_{\succ^{\prime \prime} v}=\operatorname{st}\left(W_{\succ w}, \mathbf{X}\right) \cup \operatorname{st}(w, \mathbf{X})_{\succ}{ }^{\prime \prime} v$. If, $w=\hat{w}$, then $\mathbf{X}_{\succ{ }^{\prime \prime} v}=$ $\operatorname{st}(w, \mathbf{X})_{\succ}{ }^{\prime \prime} v$. 
Proof. If $w \neq \hat{w}$, then $\operatorname{st}\left(W_{\succ w}, \mathbf{X}\right)=\mathbf{X}_{\succ^{\prime} w}=\mathbf{X}_{\succ^{\prime \prime} w}$ by Lemma 6 . Therefore it is sufficient to show that every $\sigma \in \mathbf{X}_{\succ}{ }^{\prime \prime} v$ which contains a vertex $v^{\prime}$ with $v^{\prime} \preceq w$ belongs to $\operatorname{st}(w, \mathbf{X})_{\succ}{ }^{\prime \prime}$. This will resolve both cases, $w=\hat{w}$ and $w \neq \hat{w}$, simultaneously. The ideas in the reminder of the proof are very similar to the ideas in the proof of Claim 7.3 .

First, we check that $\sigma \in \operatorname{st}\left(w^{\prime}, \mathbf{X}\right)$ for some $w^{\prime} \succeq w$. If $w$ is the first vertex of $W$, then this follows from the fact that $W$ induces a star decomposition of $\mathbf{X}$. If $w$ is not the first vertex of $W$, let $w^{-}$be the vertex of $W$ that immediately precedes $w$. By Lemma $6, \operatorname{st}\left(W_{\succ w^{-}}, \mathbf{X}\right)=\mathbf{X}_{\succ}{ }_{w^{-}}$. Because $\sigma \in \mathbf{X}_{\succ \prime \prime} w^{-}$, this implies that there is $w^{\prime} \succ w^{-}$with $\sigma \in \operatorname{st}\left(w^{\prime}, \mathbf{X}\right)$.

On the other hand, $\sigma$ cannot belong to $\operatorname{st}\left(w^{\prime \prime}, \mathbf{X}\right)$ with $w^{\prime \prime} \succ w$ as $\sigma$ contains $v^{\prime}$ with $v^{\prime} \preceq w$. Therefore, $w^{\prime}=w$. Given that $\operatorname{st}(w, \mathbf{X})_{\succ^{\prime \prime} v}=\mathbf{X}_{\succ^{\prime \prime} v} \cap \operatorname{st}(w, \mathbf{X})$, we deduce that $\sigma \in \operatorname{st}(w, \mathbf{X})_{\succ}{ }^{\prime} v$.

The union of two pure $k$-dimensional complexes is a pure $k$-dimensional complex. Therefore, due to Claim 7.5, it remains to check that $\operatorname{st}\left(W_{\succ w}, \mathbf{X}\right)$ and $\operatorname{st}(w, \mathbf{X})_{\succ}{ }^{\prime \prime} v$ are pure $k$-dimensional (the former case applies only if $w \neq \hat{w}$ ).

Checking that $\operatorname{st}\left(W_{\succ w}, \mathbf{X}\right)$ is pure $k$-dimensional is easy; see Remark 4 (ii).

For checking that $\operatorname{st}(w, \mathbf{X})_{\succ^{\prime \prime} v}$ is pure $k$-dimensional, we need that $\operatorname{lk}(w, \mathbf{X})_{\succ^{\prime \prime} v}$ is pure $(k-1)$-dimensional. Because $v \in P(w), \operatorname{lk}(w, \mathbf{X})_{\succ^{\prime \prime} v}=\operatorname{lk}(w, \mathbf{X})_{\unrhd \prime \prime}$ where $\triangleleft^{\prime \prime}$ is the shedding of $\operatorname{lk}(w, \mathbf{X})$ as introduced below Claim 7.1. This means that $\operatorname{lk}(w, \mathbf{X})_{\succ}{ }^{\prime \prime} v$ is an intermediate step in the shedding $\unrhd^{\prime \prime}$ of $l \mathrm{k}(w, \mathbf{X})$. If we realize that $v$ is not among the last $k$ vertices of the order $\triangleleft^{\prime \prime}$ on $\operatorname{lk}(w, \mathbf{X})$, then we can deduce that $\operatorname{lk}(w, \mathbf{X})_{\succ}{ }^{\prime \prime}$ is pure and $(k-1)$-dimensional.

If $w \neq \hat{w}$, then $\operatorname{lk}(w, \mathbf{X})_{\succ}{ }^{\prime \prime} v$ still contains the overlap $\mathbf{O}\left(w, W_{\succ} w\right)$ which is $(k-1)$-dimensional by Remark 4(ii). If $w=\hat{w}$, then we assume that $v$ is not among the last $k+1$ vertices of $\prec$ " while $\prec^{\prime \prime}$ and $\triangleleft^{\prime \prime}$ coincide on $P(\hat{w})$ and the vertices of $P(\hat{w})$ immediately precede $\hat{w}$ in $\prec$. This finishes Case 2 and thereby the proof of the theorem.

\section{$5 \quad$ Star decomposability in vertices}

Star decomposability of a barycentric subdivision. In our approach, we will need to consider the star decomposability of the barycentric subdivision $\operatorname{sd}(\mathbf{X})$ of a complex $\mathbf{X}$. In fact, we will consider only a special type of star decomposition of $\operatorname{sd}(\mathbf{X})$ using only stars of vertices of $\mathbf{X}$, that is, the faces of $\mathbf{X}$ which are actually vertices of $X$. For a well working induction, we will need that this property is kept also in the link condition and the last vertex condition of Definition 3. For stating this precisely, first, we need a more explicit description of $\operatorname{lk}(\vartheta, \operatorname{sd}(\mathbf{X}))$ if $\vartheta$ is a face (possibly a vertex) of $\mathbf{X}$.

Lemma 8. Let $\vartheta$ be a face of a simplicial complex $\mathbf{X}$, then

$$
\operatorname{lk}(\vartheta, \operatorname{sd} \mathbf{X}) \cong \operatorname{sd} \partial \vartheta * \operatorname{sdlk}(\vartheta, \mathbf{X})
$$

In particular, if $x$ is a vertex of $\mathbf{X}$, then

$$
\operatorname{lk}(x, \operatorname{sd} \mathbf{X}) \cong \operatorname{sdlk}(x, \mathbf{X}) .
$$

Proof. We will construct a simplicial isomorphism

$$
\Psi: V(\operatorname{lk}(\vartheta, \operatorname{sd} \mathbf{X})) \rightarrow V(\operatorname{sd} \partial \vartheta * \operatorname{sdlk}(\vartheta, \mathbf{X})) .
$$

First, we observe that

$$
V(\operatorname{sd} \partial \vartheta * \operatorname{sdlk}(\vartheta, \mathbf{X}))=V(\operatorname{sd} \partial \vartheta) \sqcup V(\operatorname{sdlk}(\vartheta, \mathbf{X}))=\partial \vartheta \sqcup \operatorname{lk}(\vartheta, \mathbf{X}) .
$$




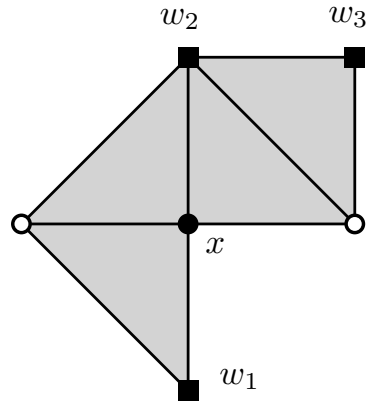

$\mathbf{X}$

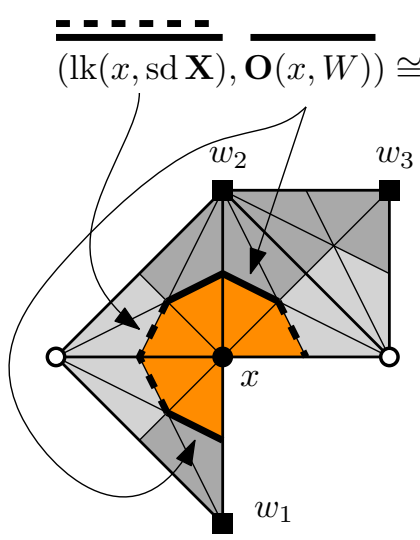

sd $\mathbf{X}$

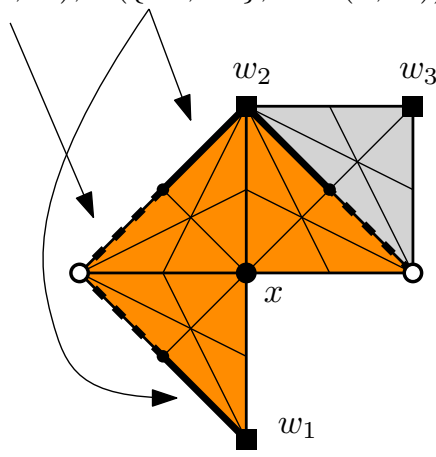

sd $\mathbf{X}$

Figure 10: Isomorphism from Lemma 9 with $W=\left\{w_{1}, w_{2}, w_{3}\right\}$. The left hand side of the formula in Lemma 9 is depicted in the middle picture and the right hand side is in the right picture. Note that $W \cap V(\operatorname{lk}(x, \mathbf{X}))=\left\{w_{1}, w_{2}\right\}$ as $w_{3}$ does not belong to $\operatorname{lk}(x, \mathbf{X})$.

Next, we realize that the vertices of $\operatorname{lk}(\vartheta, \mathrm{sd} \mathbf{X})$ are all the faces $\lambda \neq \emptyset, \vartheta$ of $\mathbf{X}$ such that $\{\lambda, \vartheta\}$ forms a simplex of sd $\mathbf{X}$, that is, either $\emptyset \neq \lambda \subsetneq \vartheta$ or $\vartheta \subsetneq \lambda$. Thus, we can define $\Psi$ in the following way

$$
\Psi(\lambda)= \begin{cases}\lambda \in \partial \vartheta & \text { if } \emptyset \neq \lambda \subsetneq \vartheta, \\ \lambda \backslash \vartheta \in \operatorname{lk}(\vartheta, \mathbf{X}) & \text { if } \vartheta \subsetneq \lambda .\end{cases}
$$

From the description above, it immediately follows that $\Psi$ is a bijection. It is also routine to check that $\Psi$ is a simplicial isomorphism. Indeed, a $\operatorname{simplex}$ of $l \mathrm{k}(\vartheta, \mathrm{sd} \mathbf{X})$ is a collection $\left\{\alpha_{1}, \ldots, \alpha_{k}, \beta_{1}, \ldots, \beta_{\ell}\right\}$ satisfying

$$
\emptyset \neq \alpha_{1} \subsetneq \cdots \subsetneq \alpha_{k} \subsetneq \vartheta \subsetneq \beta_{1} \subsetneq \cdots \subsetneq \beta_{\ell} .
$$

Such a simplex maps to a simplex $\left\{\alpha_{1}, \ldots, \alpha_{k}, \beta_{1} \backslash \vartheta, \ldots, \beta_{\ell} \backslash \vartheta\right\}$ of $\operatorname{sd} \partial \vartheta * \operatorname{sd} \operatorname{lk}(\vartheta, \mathbf{X})$ and the inverse map works analogously (note that $\beta_{i} \backslash \vartheta$ is disjoint from $\vartheta$ whereas $\alpha_{i}$ are subsets of $\vartheta)$.

Now, we extend the isomorphism above to certain pairs; for the statement, recall that $\mathbf{O}(x, W)$ is defined via formula (1).

Lemma 9. Let $x$ be a vertex and $W$ a subset of vertices of the simplicial complex $\mathbf{X}$ such that $x \notin W$. Then

$$
\left(\operatorname{lk}(x, \operatorname{sd} \mathbf{X}), \mathbf{O}_{\mathrm{sd}} \mathbf{X}(x, W)\right) \cong(\operatorname{sdlk}(x, \mathbf{X}), \operatorname{st}(W \cap V(\operatorname{lk}(x, \mathbf{X})), \operatorname{sdlk}(x, \mathbf{X}))) .
$$

Though the formula in Lemma 9 may seem complicated at first sight, it has a nice geometric interpretation. All objects are subcomplexes of sd $\mathbf{X}$ and the isomorphism in the formula pushes the pair on the left hand side farther away from $x$; see Figure 10.

Proof. From Lemma 8 we have a simplicial isomorphism $\Psi$ from $\operatorname{lk}(x, \operatorname{sd} \mathbf{X})$ to $\operatorname{sd} \operatorname{lk}(x, \mathbf{X})$. Therefore, it remains to show that $\Psi$ maps $\mathbf{O}_{\text {sd }} \mathbf{X}(x, w):=\operatorname{lk}(x, \operatorname{sd} \mathbf{X}) \cap \operatorname{lk}(w, \operatorname{sd} \mathbf{X})$ to st $(w, \operatorname{sd} \operatorname{lk}(x, \mathbf{X}))$ for $w \in W \cap V(\operatorname{lk}(x, \mathbf{X}))$, where we use the explicit $\Psi$ from the proof of Lemma 8 , and that $\mathbf{O}_{\mathrm{sd}} \mathbf{X}(x, w)=\emptyset$ for $w \in W \backslash V(\operatorname{lk}(x, \mathbf{X}))$. (Note that $\mathbf{O}_{\mathrm{sd}} \mathbf{X}(x, W)=\bigcup_{w \in W} \mathbf{O}_{\mathrm{sd}} \mathbf{X}(x, w)$.) 
The faces of $\mathbf{O}_{\mathrm{sd}} \mathbf{X}(x, w)$ are collections $\left\{\beta_{1}, \ldots, \beta_{\ell}\right\}$ of faces of $\mathbf{X}$ satisfying

$$
\{x, w\} \subseteq \beta_{1} \subsetneq \cdots \subsetneq \beta_{\ell} .
$$

Let us emphasize that the first inclusion need not be strict. Therefore, $\mathbf{O}_{\mathrm{sd} X}(x, w)$ is non-empty if and only if $\{x, w\} \in \mathbf{X}$, that is, if and only if $w \in W \cap V(\operatorname{lk}(x, \mathbf{X}))$ as required. In sequel, we assume that $w \in W \cap V(\operatorname{lk}(x, \mathbf{X}))$.

The collections $\left\{\beta_{1}, \ldots, \beta_{\ell}\right\}$ are mapped under $\Psi$ to $\left\{\beta_{1} \backslash\{x\}, \ldots, \beta_{\ell} \backslash\{x\}\right\}$ satisfying the same condition due to the description of $\Psi$ in the proof of Lemma 8 . Setting $\gamma_{j}=\beta_{j} \backslash\{x\}$ we get

$$
\{w\} \subseteq \gamma_{1} \subsetneq \cdots \subsetneq \gamma_{\ell}
$$

for $\gamma_{j}$ not containing $x$ but such that $\gamma_{j} \cup\{x\}$ is a face of $\mathbf{X}$, which is exactly a description of $\operatorname{st}(w, \operatorname{sd}(\operatorname{lk}(x, \mathbf{X})))$.

Now, we can define star decomposibility in vertices:

Definition 10 (Star decomposability in vertices). Let $\mathbf{X}$ be a pure, $k$-dimensional simplicial complex, $k \geq-1$ and let $X \subseteq V(\mathbf{X})$. We inductively define star decomposability in vertices of the pair $(\operatorname{sd} \mathbf{X}, X)$. We also say that $\mathrm{sd} \mathbf{X}$ is star decomposable in vertices if the pair $(\operatorname{sd} \mathbf{X}, V(\mathbf{X})$ ) is star decomposable in vertices.

If $k=-1$, then $(\operatorname{sd}\{\emptyset\}, \emptyset)=(\{\emptyset\}, \emptyset)$ is star decomposable in vertices. (This is the same as star decomposability in this case.)

If $k \geq 0$, then $(\operatorname{sd} \mathbf{X}, X)$ is star decomposable in vertices, if there is a total order $\prec$ on the set $V(\mathbf{X})$, inducing a star partition of sd $\mathbf{X}$, with the following properties $]^{7}$

Order condition: $X=V(\mathbf{X})_{\succeq w^{\prime}}$ for some $w^{\prime} \in V(\mathbf{X})$.

Link condition: For any vertex $w \in V(\mathbf{X})$ except for the last vertex in the order $\prec$, the pair $\left(\operatorname{sdlk}(w, \mathbf{X}), V(\operatorname{lk}(w, \mathbf{X}))_{\succ w}\right)$ is star decomposable in vertices.

Last vertex condition: For the last vertex $\hat{x} \in V(\mathbf{X})$ in the order $\prec$, the link $\operatorname{sd} \operatorname{lk}(\hat{x}, \mathbf{X})$ is star decomposable in vertices.

If the order $\prec$ on $W$ satisfies the three conditions above, we say that $\prec$ induces a star decomposition of $(\mathrm{sd} \mathbf{X}, X)$ in vertices.

Lemma 9 implies the following proposition.

Proposition 11. Let us assume that the pair $(\mathrm{sd} \mathbf{X}, X)$ is star decomposable in vertices, then it is star decomposable.

Proof. We check that the order condition, the link condition and the last vertex condition in Definition 3 imply the corresponding conditions in Definition 10. The rest of the proof is a straightforward induction given that in dimensions -1 and 0 the notions coincide.

The order condition in Definitions 3 and 10 is actually identical.

For checking the link condition in Definition 3, for a given $w \in V(\mathbf{X})$ we need to find a set $U \subseteq V(\operatorname{lk}(w, \operatorname{sd} \mathbf{X}))$ such that (i) $\operatorname{st}(U, \operatorname{lk}(w, \operatorname{sd} \mathbf{X}))=\mathbf{O}_{\mathrm{sd}} \mathbf{X}\left(w, V(\mathbf{X})_{\succ w}\right)$ and (ii) the pair $(\operatorname{lk}(w, \operatorname{sd} \mathbf{X}), U)$ is star decomposable in vertices (therefore star decomposable by induction). By Lemma 9 we have an isomorphism $\Psi$ mapping the pair

$$
\left(\operatorname{lk}(w, \operatorname{sd} \mathbf{X}), \mathbf{O}_{\mathrm{sd}} \mathbf{X}\left(w, V(\mathbf{X})_{\succ w}\right)\right)
$$

\footnotetext{
${ }^{7}$ Note that $V(\mathbf{X})$ induces a star partition of sd $\mathbf{X}$ for an arbitrary complex $\mathbf{X}$.
} 
to the pair

$$
\left(\operatorname{sdlk}(w, \mathbf{X}), \operatorname{st}\left(V(\operatorname{lk}(w, \mathbf{X}))_{\succ w}, \operatorname{sdlk}(w, \mathbf{X})\right)\right),
$$

using that $V(\mathbf{X})_{\succ w} \cap V(\operatorname{lk}(w, \mathbf{X}))=V(\operatorname{lk}(w, \mathbf{X}))_{\succ w}$. We set $U:=\Psi^{-1}\left(V(\operatorname{lk}(w, \mathbf{X}))_{\succ w}\right)$, then (i) follows immediately from the isomorphism above. On the other hand, $(\operatorname{lk}(w, \operatorname{sd} \mathbf{X}), U)$ is isomorphic to $\left(\operatorname{sdlk}(w, \mathbf{X}), V(\operatorname{lk}(w, \mathbf{X}))_{\succ w}\right)$ by applying $\Psi$. Therefore, (ii) indeed follows from the link condition of Definition 10 .

Finally the last vertex condition of Definition 10 implies the same condition of Definition 3 via Lemma 8 (and the induction).

Merging orders inducing a star decomposition in vertices. Given simplicial complexes $\mathbf{X}$ and $\mathbf{Y}$ such that $\operatorname{sd}(\mathbf{X})$ and $\operatorname{sd}(\mathbf{Y})$ are star decomposable in vertices, we want to provide an order on $V(\mathbf{X}) \sqcup V(\mathbf{Y})$ which induces a star decomposition in vertices of $\operatorname{sd}(\mathbf{X} * \mathbf{Y})$. For the proof of our main result we need some flexibility how to merge the orders on $V(\mathbf{X})$ and $V(\mathbf{Y})$. First we provide a recipe that works in general but does not give all we need. This is the contents of forthcoming Proposition 12. Then we also provide a more specific recipe which gives more under additional assumptions on $\mathbf{Y}$ (see Proposition 14).

Proposition 12. Let $\mathbf{X}$ and $\mathbf{Y}$ be pure simplicial complexes such that $\operatorname{sd}(\mathbf{X})$ and $\operatorname{sd}(\mathbf{Y})$ are star decomposable in vertices. Let $\prec$ be an arbitrary total order on $V(\mathbf{X}) \sqcup V(\mathbf{Y})$ satisfying that

(i) the restriction of $\prec$ to $V(\mathbf{X})$ induces a star decomposition in vertices of $\operatorname{sd}(\mathbf{X})$,

(ii) the restriction of $\prec$ to $V(\mathbf{Y})$ induces a star decomposition in vertices of $\operatorname{sd}(\mathbf{Y})$,

(iii) if both $\mathbf{X}$ and $\mathbf{Y}$ are nonempty, then the last two elements in $\prec$ are the last element of $V(\mathbf{X})$ and the last element of $V(\mathbf{Y})$ (in arbitrary order).

Then $\operatorname{sd}(\mathbf{X} * \mathbf{Y})$ is star decomposable in vertices in the order $\prec$ on $V(\mathbf{X} * \mathbf{Y})=V(\mathbf{X}) \sqcup V(\mathbf{Y})$.

Corollary 13. Let $\mathbf{X}$ and $\mathbf{Y}$ be simplicial complexes and $X \subseteq V(\mathbf{X}), Y \subseteq V(\mathbf{Y})$. Assume that the pairs $(\mathrm{sd} \mathbf{X}, X)$ and $(\mathrm{sd} \mathbf{Y}, Y)$ are star decomposable in vertices. Then the pair $(\operatorname{sd}(\mathbf{X} *$ $\mathbf{Y}), X \sqcup Y)$ is star decomposable in vertices as well. In addition, if $|Y|=1$, then the pair $(\operatorname{sd}(\mathbf{X} * \mathbf{Y}), Y)$ is star decomposable in vertices.

Proof of Corollary 13. First, let us assume that $X=\emptyset$. Because $(\operatorname{sd} \mathbf{X}, X)$ is star decomposable, we deduce that $\mathbf{X}=\{\emptyset\}$. Consequently, $(\operatorname{sd}(\mathbf{X} * \mathbf{Y}), X \sqcup Y)=(\operatorname{sd} \mathbf{Y}, Y)$, which is star decomposable in vertices. Similarly, we resolve the case $Y=\emptyset$.

Now we can assume $X, Y \neq \emptyset$. Let $\prec \mathbf{x}$ be a total order on $V(\mathbf{X})$ inducing a star decomposition of ( $\operatorname{sd} \mathbf{X}, X)$ in vertices and let $\prec \mathbf{Y}$ be a total order on $V(\mathbf{Y})$ inducing a star decomposition of $(\operatorname{sd} \mathbf{Y}, Y)$ in vertices. Let $\hat{x}$ be the last vertex of $V(\mathbf{X})$ in $\prec \mathbf{X}$ and $\hat{y}$ be the last vertex of $V(\mathbf{Y})$ in $\prec \mathbf{Y}$. Necessarily, $\hat{x} \in X$ and $\hat{y} \in Y$ as $X, Y \neq \emptyset$.

We define a total order $\prec$ on $V(\mathbf{X}) \sqcup V(\mathbf{Y})$ so that we consider the vertices of $V(\mathbf{X}) \sqcup V(\mathbf{Y})$ in the order $[V(\mathbf{X}) \backslash X, V(\mathbf{Y}) \backslash Y, X \backslash\{\hat{x}\}, Y \backslash\{\hat{y}\}, \hat{x}, \hat{y}]$, where the individual sets $V(\mathbf{X}) \backslash X$, $V(\mathbf{Y}) \backslash Y, X \backslash\{\hat{x}\}$, and $Y \backslash\{\hat{y}\}$ are sorted according to $\prec \mathbf{X}$ and $\prec \mathbf{Y}$ respectively. Then $\prec$ satisfies the assumptions of Proposition 12 . Therefore, $\operatorname{sd}(\mathbf{X} * \mathbf{Y})$ is star decomposable in vertices in the order $\prec$.

Given that $\operatorname{st}(X \sqcup Y, \operatorname{sd}(\mathbf{X} * \mathbf{Y}))=\operatorname{st}\left((V(\mathbf{X}) \sqcup V(\mathbf{Y}))_{\succeq z}, \operatorname{sd}(\mathbf{X} * \mathbf{Y})\right)$ where $z$ is the first vertex of $X \cup Y$ in $\prec$, we deduce that $\prec$ gives also a star decomposition of $(\operatorname{sd}(\mathbf{X} * \mathbf{Y}), X \sqcup Y)$ in vertices.

Finally, if $|Y|=1$, then $Y=\{\hat{y}\}$. Thus $\operatorname{st}(Y, \operatorname{sd}(\mathbf{X} * \mathbf{Y}))=\operatorname{st}\left((V(\mathbf{X}) \sqcup V(\mathbf{Y}))_{\succeq \hat{y}}, \operatorname{sd}(\mathbf{X} * \mathbf{Y})\right)$ which means that $\prec$ gives a star decomposition of $(\operatorname{sd}(\mathbf{X} * \mathbf{Y}), Y)$ in vertices as well. 
Proof of Proposition 12. First, similarly as in the previous proof, the statement is trivial if $\mathbf{X}=\{\emptyset\}$ or $\mathbf{Y}=\{\emptyset\}$ as a join with $\{\emptyset\}$ yields the same complex. Therefore, we can assume $\mathbf{X}, \mathbf{Y} \neq\{\emptyset\}$. In particular, the item (iii) of the statement is non-void.

Now, we prove the proposition by induction on $\operatorname{dim}(\mathbf{X} * \mathbf{Y})$. The start of the induction, when $\operatorname{dim}(\mathbf{X} * \mathbf{Y}) \leq 0$, is covered by the observation above.

We are given the order $\prec$ on $V(\mathbf{X} * \mathbf{Y})$; therefore it remains to check the order condition, the link condition and the last vertex condition.

As we check star decomposability of $\operatorname{sd}(\mathbf{X} * \mathbf{Y})$, that is, the pair $(\operatorname{sd}(\mathbf{X} * \mathbf{Y}), V(\mathbf{X}) \sqcup V(\mathbf{Y}))$, the order condition is trivial. (It is sufficient to take the first vertex of $V(\mathbf{X}) \sqcup V(\mathbf{Y})$ for checking the order condition.)

For checking the link condition, we consider arbitrary $x \in V(\mathbf{X}) \sqcup V(\mathbf{Y})$ distinct from the last vertex. Without loss of generality, we can assume $x \in V(\mathbf{X})$ as the argument is symmetric for a vertex from $V(\mathbf{Y})$. We need to check star decomposability of the pair

$$
\left(\operatorname{sd}(\operatorname{lk}(x, \mathbf{X} * \mathbf{Y})), V(\operatorname{lk}(x, \mathbf{X} * \mathbf{Y}))_{\succ x}\right) .
$$

Given that $x \in V(\mathbf{X})$, this equals

$$
\left(\operatorname{sd}(\operatorname{lk}(x, \mathbf{X}) * \mathbf{Y}),(V(\operatorname{lk}(x, \mathbf{X})) \sqcup V(\mathbf{Y}))_{\succ x}\right) .
$$

From the assumption on star decomposability of $\operatorname{sd} \mathbf{Y}$ in the order $\prec$, we deduce that the pair

$$
\left(\operatorname{sd}(\mathbf{Y}), V(\mathbf{Y})_{\succ x}\right)
$$

is star decomposable in vertices as long as $V(\mathbf{Y})_{\succ x}$ is nonempty. However, $V(\mathbf{Y})_{\succ x}$ is indeed nonempty as $x$ is not the last vertex of $V(\mathbf{X}) \sqcup V(\mathbf{Y})$ in $\prec$ whereas there is a vertex from $V(\mathbf{Y})$ among the last two vertices.

From the assumption on star decomposability of $\mathbf{X}$ in the order $\prec$, checking the link condition gives that the pair

$$
\left(\operatorname{sdlk}(x, \mathbf{X}), V(\operatorname{lk}(x, \mathbf{X}))_{\succ x}\right)
$$

is star decomposable in vertices if $x$ is not the last vertex of $V(\mathbf{X})$. Therefore, if $x$ is not the last vertex of $V(\mathbf{X})$, we will use the induction. From Corollary 13 for pairs (6) and (5) we deduce that the pair in (4) is indeed star decomposable in vertices as required. (Note that this is a correct use of the induction as we deduced Corollary 13 from Proposition 12 in the same dimension.)

It remains to consider the case when $x$ is a last vertex of $V(\mathbf{X})$. In this case, $x$ is the second to last vertex of $V(\mathbf{X}) \sqcup V(\mathbf{Y})$. Let $\hat{y}$ be the last vertex of $V(\mathbf{Y})$, that is, the last vertex of $V(\mathbf{X}) \sqcup V(\mathbf{Y})$ as well. Then the pair (4) simplifies to

$$
(\operatorname{sd}(\operatorname{lk}(x, \mathbf{X}) * \mathbf{Y}),\{\hat{y}\}) .
$$

Now, we can use Corollary 13 again with pairs $(\operatorname{sdlk}(x, \mathbf{X}), V(\operatorname{lk}(x, \mathbf{X})))$ and $(\operatorname{sd}(\mathbf{Y}),\{\hat{y}\})$, using the 'in addition' part.

Finally, it remains to check the last vertex condition. Let us therefore assume that $\hat{x}$ is the last vertex of $V(\mathbf{X}) \sqcup V(\mathbf{Y})$. Again, we can without loss of generality assume that $\hat{x} \in V(\mathbf{X})$. We need to check star decomposability in vertices of $\operatorname{sdlk}(\hat{x}, \mathbf{X} * \mathbf{Y})=\operatorname{sd}(\operatorname{lk}(\hat{x}, \mathbf{X}) * \mathbf{Y})$. By the last vertex condition on $\operatorname{sd}(\mathbf{X})$ we get that $\operatorname{sdlk}(\hat{x}, \mathbf{X})$ is star decomposable in vertices. Therefore, by the induction applied to $\operatorname{sd}(\operatorname{lk}(\hat{x}, \mathbf{X}))$ and $\operatorname{sd} \mathbf{Y}$, we get that $\operatorname{sd}(\operatorname{lk}(\hat{x}, \mathbf{X}) * \mathbf{Y})$ is star decomposable in vertices as required. 
Now, we state a more specialized version of Proposition 12 with an additional condition on homology. Let us recall that given a simplicial complex $\mathbf{Y}$ and $Y \subseteq V(\operatorname{sd} \mathbf{Y})$, the $\operatorname{star} \operatorname{st}(Y, \operatorname{sd} \mathbf{Y})$ is defined as $\bigcup_{v \in Y} \operatorname{st}(v, \mathrm{sd} \mathbf{Y})$. Following our convention of neglecting a difference between $v \in V(\mathbf{Y})$ and $\{v\} \in V(\operatorname{sd} \mathbf{Y})$, we also set $\operatorname{st}(Y, \operatorname{sd} \mathbf{Y}):=\bigcup_{v \in Y} \operatorname{st}(v, \operatorname{sd} \mathbf{Y})$ for $Y \subseteq V(\mathbf{Y})$.

Proposition 14. Let $\mathbf{X}$ and $\mathbf{Y}$ be pure simplicial complexes, $\operatorname{dim} \mathbf{X}, \operatorname{dim} \mathbf{Y} \geq 0$, and $Y$ be a nonempty subset of $V(\mathbf{Y})$. Assume that $\mathrm{sd} \mathbf{X}$ and $(\mathrm{sd} \mathbf{Y}, Y)$ are star decomposable in vertices and $\operatorname{st}(Y, \operatorname{sd} \mathbf{Y})$ has trivial reduced homology groups. Let $\prec$ be an arbitrarily total order on $V(\mathbf{X}) \sqcup V(\mathbf{Y})$ satisfying:

(i) The restriction of $\prec$ to $V(\mathbf{X})$ induces a star decomposition in vertices of $\operatorname{sd}(\mathbf{X})$;

(ii) The restriction of $\prec$ to $V(\mathbf{Y})$ induces a star decomposition in vertices of $\operatorname{sd}(\mathbf{Y}, Y)$; and

(iii) $Y=(V(\mathbf{X}) \sqcup V(\mathbf{Y}))_{\succ \hat{x}}$ where $\hat{x}$ is the last vertex of $V(\mathbf{X})$ in $\prec$.

Then $\operatorname{sd}(\mathbf{X} * \mathbf{Y}, Y)$ is star decomposable in vertices in the order $\prec$ on $V(\mathbf{X} * \mathbf{Y})=V(\mathbf{X}) \sqcup V(\mathbf{Y})$.

For the proof, we need a following auxiliary lemma which will be useful in the induction.

Lemma 15. Let $\mathbf{Y}$ be a pure simplicial complex and $Y \subseteq V(\mathbf{Y})$. Assume that the pair $(\operatorname{sd} \mathbf{Y}, Y)$ is star-decomposable in vertices in some total order $\prec$ on $V(\mathbf{Y})$ and that $\operatorname{st}(Y, \operatorname{sd} \mathbf{Y})$ has trivial reduced homology groups. Then $\operatorname{st}\left(V(\operatorname{lk}(y, \mathbf{Y}))_{\succ y}, \operatorname{sd}(\mathrm{lk}(y, \mathbf{Y}))\right)$ has trivial reduced homology groups as well for all $y \in Y$ except for the last vertex in $Y$.

Proof. Let $y \in Y$ be different from the last vertex in the order $\prec$. First, we show that $\operatorname{st}\left(Y_{\succ y}, \mathbf{Y}\right)$ has trivial reduced homology groups.

Since the pair $(\operatorname{sd} \mathbf{Y}, Y$ ) is star decomposable in vertices, Theorem 7 implies that $\operatorname{sd} \mathbf{Y}$ is vertex decomposable. In addition, we get that sd $\mathbf{Y}$ is vertex decomposable in a shedding order $\succ^{\prime \prime}$ extending $\succ^{\prime}$ where is derived from $\succ$. (We recall that the definition of the derived order is given above the statement of Lemma 6.) In particular, $\operatorname{st}(Y, \operatorname{sd} \mathbf{Y})$ and later $\operatorname{st}\left(Y_{\succ y}, \operatorname{sd} \mathbf{Y}\right)$ are intermediate steps in the sequence of complexes obtained by gradually removing vertices of $\mathbf{Y}$ in the given shedding order $\succ^{\prime \prime}$.

We also get that $\operatorname{st}(Y, \operatorname{sd} \mathbf{Y})$ and $\operatorname{st}\left(Y_{\succ y}, \mathrm{sd} \mathbf{Y}\right)$ are shellable by PB80 (see Theorem 2.8 and the note below Definition 2.1 in [PB80]). Therefore, each of them is homotopy equivalent to a wedge of $d$-spheres where $d=\operatorname{dim} \mathbf{Y}$; see [Koz08, Theorem 12.3]. Since $\operatorname{st}(Y, \operatorname{sd} \mathbf{Y})$ has trivial homology groups, this must be a trivial wedge. However, following the shedding order from $\operatorname{st}(Y, \operatorname{sd} \mathbf{Y})$ to $\operatorname{st}\left(Y_{\succ y}, \mathrm{sd} \mathbf{Y}\right)$, we cannot introduce homology in dimension $d$ when gradually removing vertices. Therefore, $\operatorname{st}\left(Y_{\succ y}, \mathrm{sd} \mathbf{Y}\right)$ has to be homotopy equivalent to a trivial wedge as well showing that $\operatorname{st}\left(Y_{\succ y}\right.$, sd $\left.\mathbf{Y}\right)$ has trivial reduced homology groups.

Note that $\operatorname{st}\left(Y_{\succeq y}, \mathrm{sd} \mathbf{Y}\right)$ has trivial reduced homology groups as well by analogous reasoning.

Now, by Lemma 9

$$
\operatorname{st}\left(V(\operatorname{lk}(y, \mathbf{Y}))_{\succ y}, \operatorname{sd}(\operatorname{lk}(y, \mathbf{Y}))\right) \cong \mathbf{O}_{\mathrm{sd}} \mathbf{Y}\left(y, V(\mathbf{Y})_{\succ y}\right) .
$$

We use a Mayer-Vietoris sequence for $\operatorname{st}\left(Y_{\succeq y}, \operatorname{sd} \mathbf{Y}\right)$ covered by $\operatorname{st}(y, \operatorname{sd}(\mathbf{Y}))$ and $\operatorname{st}\left(Y_{\succ y}, \operatorname{sd} \mathbf{Y}\right)$. Then $\operatorname{st}(y, \operatorname{sd}(\mathbf{Y})) \cap \operatorname{st}\left(Y_{\succ y}, \operatorname{sd} \mathbf{Y}\right)=\mathbf{O}_{\text {sd } \mathbf{Y}}\left(y, Y_{\succ y}\right)=\mathbf{O}_{\text {sd } \mathbf{Y}}\left(y, V(\mathbf{Y})_{\succ y}\right)$ and we get the following long exact sequence

$$
\begin{aligned}
\cdots & \longrightarrow \tilde{H}_{n+1}\left(\operatorname{st}\left(Y_{\succeq y}, \operatorname{sd} \mathbf{Y}\right)\right) \longrightarrow \tilde{H}_{n}\left(\mathbf{O}_{\text {sd } \mathbf{Y}}\left(y, V(\mathbf{Y})_{\succ y}\right)\right) \longrightarrow \\
& \longrightarrow \tilde{H}_{n}(\operatorname{st}(y, \operatorname{sd} \mathbf{Y})) \oplus \tilde{H}_{n}\left(\operatorname{st}\left(Y_{\succ y}, \operatorname{sd} \mathbf{Y}\right)\right) \longrightarrow \tilde{H}_{n}\left(\operatorname{st}\left(Y_{\succeq y}, \operatorname{sd} \mathbf{Y}\right)\right) \longrightarrow \cdots
\end{aligned}
$$

All st $\left(Y_{\succeq y}, \operatorname{sd} \mathbf{Y}\right), \operatorname{st}(y, \operatorname{sd} \mathbf{Y})$ and $\operatorname{st}\left(Y_{\succ y}, \operatorname{sd} \mathbf{Y}\right)$ have trivial reduced homology groups. Therefore, $\tilde{H}_{n}\left(\mathbf{O}_{\mathrm{sd}} \mathbf{Y}\left(y, V(\mathbf{Y})_{\succ y}\right)\right) \cong \tilde{H}_{n}\left(\operatorname{st}\left(V(\operatorname{lk}(y, \mathbf{Y}))_{\succ y}, \operatorname{sd}(\operatorname{lk}(y, \mathbf{Y}))\right)\right.$ is trivial for all $n \in \mathbb{Z}$. 
Proof of Proposition 14. Similarly, as in the proof of Proposition 12, we proceed by induction on $\operatorname{dim}(\mathbf{X} * \mathbf{Y})$.

First, we observe that the case $\operatorname{dim} \mathbf{Y}=0$ is covered by Proposition 12 . Indeed, the only issue is to verify (iii) of Proposition 12. If $\operatorname{dim} \mathbf{Y}=0$, then $Y$ must contain a single vertex $\hat{y}$ (due to the condition on homology of $\operatorname{st}(Y, \operatorname{sd}(\mathbf{Y}))$ ). Consequently, (iii) (of this proposition) implies that the last two vertices of $\prec$ are $\hat{x}$ and $\hat{y}$ which verifies (iii) of Proposition 12 .

Now, let us assume $\operatorname{dim} \mathbf{X} \geq 0$ and $\operatorname{dim} \mathbf{Y} \geq 1$. The order condition is satisfied since $Y$ is non-empty and it is equal to $(V(\mathbf{X}) \sqcup V(\mathbf{Y}))_{\succ \hat{x}}$ by (iii).

For checking the link condition, we consider arbitrary $z \in V(\mathbf{X}) \sqcup V(\mathbf{Y})$ distinct from the last vertex. We need to check star decomposability of the pair

$$
\left(\operatorname{sd}(\operatorname{lk}(z, \mathbf{X} * \mathbf{Y})), V(\operatorname{lk}(z, \mathbf{X} * \mathbf{Y}))_{\succ z}\right) .
$$

If $z \in V(\mathbf{X}) \backslash\{\hat{x}\} \sqcup V(\mathbf{Y}) \backslash Y$, then the analysis is the same as in the proof of Proposition 12 . If $z=\hat{x}$, the pair (7) becomes

$$
(\operatorname{sd}(\operatorname{lk}(\hat{x}, \mathbf{X}) * \mathbf{Y}), Y) .
$$

If $\operatorname{dim} \mathbf{X}=0$, then we further get $(\operatorname{sd} \mathbf{Y}, Y)$ which is star decomposable in vertices by the assumptions. If $\operatorname{dim} \mathbf{X} \geq 1$, then $\operatorname{dim} \operatorname{lk}(\hat{x}, \mathbf{X}) \geq 0$ and we can use the induction (note that $\operatorname{sd} \operatorname{lk}(\hat{x}, \mathbf{X})$ is star decomposable in vertices by the last vertex condition for decomposition of $\operatorname{sd} \mathbf{X})$.

Finally, by assuming $z \in Y \backslash\{\hat{y}\}$, where $\hat{y}$ is the last vertex of $\prec$, we get the pair

$$
\left(\operatorname{sd}(\mathbf{X} * \operatorname{lk}(z, \mathbf{Y})), V\left(\operatorname{lk}(z, \mathbf{Y})_{\succ z}\right) .\right.
$$

By Lemma $15 \operatorname{st}\left(V\left(\operatorname{lk}(z, \mathbf{Y})_{\succ z}, \operatorname{sd}(\operatorname{lk}(z, \mathbf{Y}))\right)\right.$ has trivial reduced homology groups. Therefore, (8) is star-decomposable in vertices by the induction hypothesis. (Here we use that $\operatorname{dim} \operatorname{lk}(z, \mathbf{Y}) \geq 0$ and that $\left(\operatorname{sdlk}(z, \mathbf{Y}), V\left(\operatorname{lk}(z, \mathbf{Y})_{\succ z}\right)\right)$ is star decomposable in vertices by the link condition for the decomposition of $(\operatorname{sd} \mathbf{Y}, Y)$.)

Finally, we check the last vertex condition. We need star decomposability in vertices of $\operatorname{sd} \operatorname{lk}(\hat{y}, \mathbf{X} * \mathbf{Y})$. Note that $\operatorname{lk}(\hat{y}, \mathbf{X} * \mathbf{Y})=\mathbf{X} * \operatorname{lk}(\hat{y}, \mathbf{Y})$ as both sides contain simplices of the form $\xi \cup \eta$, where $\xi \in \mathbf{X}, \eta \cup\{\hat{y}\} \in \mathbf{Y}$, and $\hat{y} \notin \eta$. Thus, we need star decomposability in vertices of $\operatorname{sd}(\mathbf{X} * \operatorname{lk}(\hat{y}, \mathbf{Y}))$. This is star decomposable in vertices by Proposition 12. (Here, we again use that $\operatorname{dim} \operatorname{lk}(\hat{y}, \mathbf{Y}) \geq 0$ and also that $\operatorname{sd} \operatorname{lk}(\hat{y}, \mathbf{Y})$ is star decomposable in vertices by the last vertex condition in the decomposition of sd $\mathbf{Y}$.)

\section{Proof of the main result}

In this section, we prove Theorem 2 which also finishes the proof of Theorem 1.

We first need two auxiliary observations that we will use in the proof.

Observation 16. The boundary of a simplex $\partial \sigma$ satisfies the $(H R C)$ condition.

Proof. We prove the observation by induction on $\operatorname{dim} \sigma$, starting with $\operatorname{dim} \sigma=0$, in which case $\partial \sigma=\emptyset$. If $\operatorname{dim} \sigma>0$, let $\sigma^{\prime} \subsetneq \sigma$. We need to check that $\operatorname{lk}\left(\sigma^{\prime}, \partial \sigma\right)$ satisfies the (RC) condition. This link is again a boundary of a simplex. If $\sigma^{\prime} \neq \emptyset$, we get a simplex of small dimension, therefore, we can use the induction. If $\sigma=\emptyset$, then $\operatorname{lk}\left(\sigma^{\prime}, \partial \sigma\right)=\partial \sigma$ which is collapsible after removing an arbitrary facet (it is a cone then). 
Observation 17. Let $\mathbf{K}$ be a collapsible complex and $w$ be an arbitrary vertex of $\mathbf{K}$. Then $\mathbf{K}$ collapses to $w$.

Proof. First, we use the well known fact that the collapses of $\mathbf{K}$ can be rearranged so that they are ordered by non-increasing dimension [Whi39, Section 3]. In particular, this means that $\mathbf{K}$ collapses to a graph $\mathbf{G}$ with $V(\mathbf{G})=V(\mathbf{K})$. This graph must be a tree as $\mathbf{K}$ is collapsible, and we can further rearrange the collapses of $\mathbf{G}$ so that $w$ is the last vertex.

Now we prove Theorem 2 by induction on the dimension of $\mathbf{K}$. We know that $\mathbf{K}$ satisfies the (RC) condition. Therefore, there are facets $\phi_{1}, \ldots, \phi_{t}$ of $\mathbf{K}$ such that $\mathbf{K}^{\prime}:=\mathbf{K}-\left\{\phi_{1}, \ldots, \phi_{t}\right\}$ is collapsible. We further consider a sequence $\left(\mathbf{K}_{1}, \ldots, \mathbf{K}_{s}\right)$ of elementary collapses of $\mathbf{K}^{\prime}$ where $\mathbf{K}^{\prime}=\mathbf{K}_{1}, \mathbf{K}_{s}$ is a vertex (denoted by $z$ ), and $\mathbf{K}_{i+1}$ arises from $\mathbf{K}_{i}$ by removing faces $\sigma_{i}$ and $\tau_{i}$ where $\sigma_{i} \subset \tau_{i}$ and $\operatorname{dim} \sigma_{i}=\operatorname{dim} \tau_{i}-1$, and $\tau_{i}$ is the unique maximal face containing $\sigma_{i}$. Then we consider the following total order $\prec$ on nonempty faces of $\mathbf{K}$, that is, vertices of sd $\mathbf{K}$ :

$$
\phi_{1} \prec \cdots \prec \phi_{t} \prec \sigma_{1} \prec \tau_{1} \prec \sigma_{2} \prec \tau_{2} \prec \cdots \prec \sigma_{s-1} \prec \tau_{s-1} \prec\{z\} .
$$

Our aim is to show that $\prec$ induces a star decomposition in vertices of $\operatorname{sd}^{2} \mathbf{K}$. This we will also use in the induction; that is, for complexes $\mathbf{L}$ of lower dimension satisfying the (HRC) condition, we assume that a sequence of removals of facets and collapses induces a star decomposition in vertices of $\operatorname{sd}^{2} \mathbf{L}$ as above. The proof is easy if $\operatorname{dim} \mathbf{K}=0$ (here no collapses are used), thus we may assume that $\operatorname{dim} \mathbf{K}>0$ and proceed with the second induction step.

There is essentially nothing to check for the order condition as we provide a total order on vertices of sd $\mathbf{K}$. Thus the only issue is to check the link condition and the last vertex condition.

In order to access the vertices of sd $\mathbf{K}$ more easily in the given order, we also give them alternate names $\omega_{1}, \ldots, \omega_{k}$ so that

$$
\left(\phi_{1}, \ldots, \phi_{t}, \sigma_{1}, \tau_{1}, \ldots, \sigma_{s-1}, \tau_{s-1},\{z\}\right)=\left(\omega_{1}, \ldots, \omega_{k}\right)
$$

where $k=t+2 s-1$. That is, $\phi_{1}=\omega_{1}, \sigma_{1}=\omega_{t+1}$, etc.

Checking the last vertex condition. Because it is easier, we check the last vertex condition first. We need to check that $\operatorname{sd} \operatorname{lk}\left(\omega_{k}, \operatorname{sd} \mathbf{K}\right)$ is star decomposable in vertices. Because $\omega_{k}$ is a vertex of $\mathbf{K}$, this complex is isomorphic to $\operatorname{sd}^{2} \operatorname{lk}\left(\omega_{k}, \mathbf{K}\right)$ by Lemma 8 . Therefore, this complex is star decomposable in vertices by induction because $\mathrm{lk}\left(\omega_{k}, \mathbf{K}\right)$ satisfies the (HRC) condition as this condition is hereditary for links.

Checking the link condition: For checking the link condition, we need to check that the pair $\left(\operatorname{sdlk}\left(\omega_{i}, \operatorname{sd} \mathbf{K}\right), V\left(\operatorname{lk}\left(\omega_{i}, \operatorname{sd} \mathbf{K}\right)\right)_{\succ \omega_{i}}\right)$ is star decomposable in vertices for $i \in\{1, \ldots, k-1\}$. For checking this condition we again need to 'simplify' this pair so that we remove the subdivision from the link. The tool for this is again Lemma 8. For the first entry it gives

$$
\operatorname{sdlk}\left(\omega_{i}, \operatorname{sd} \mathbf{K}\right) \cong \operatorname{sd}\left(\operatorname{sd} \partial \omega_{i} * \operatorname{sdlk}\left(\omega_{i}, \mathbf{K}\right)\right) .
$$

We use the specific isomorphism $\Psi$ from the proof of Lemma 8 and our next task is to describe $\left.V\left(\operatorname{lk}\left(\omega_{i}, \mathrm{sd} \mathbf{K}\right)\right)_{\succ \omega_{i}}\right)$ after applying this isomorphism.

First, we briefly describe the set $V\left(\operatorname{lk}\left(\omega_{i}, \operatorname{sd} \mathbf{K}\right)\right)_{\succ \omega_{i}}$. The vertices of $\operatorname{lk}\left(\omega_{i}, \operatorname{sd} \mathbf{K}\right)$ are the nonempty faces $\eta$ of $\mathbf{K}$ such that either $\eta \subsetneq \omega_{i}$ or $\omega_{i} \subsetneq \eta$. Therefore, the set $V\left(\operatorname{lk}\left(\omega_{i}, \operatorname{sd} \mathbf{K}\right)\right)_{\succ \omega_{i}}$ consists of faces $\eta$ as above, which in addition satisfy $\eta \succ \omega_{i}$. The isomorphism $\Psi$ from the proof of Lemma 8 maps $\eta$ again to $\eta$ if $\eta \subsetneq \omega_{i}$ and it maps $\eta$ to $\eta \backslash \omega_{i}$ if $\omega_{i} \subsetneq \eta$. Hence

$$
\Psi\left(V\left(\operatorname{lk}\left(\omega_{i}, \operatorname{sd} \mathbf{K}\right)\right)_{\succ \omega_{i}}\right)=V\left(\operatorname{sd} \partial \omega_{i}\right)_{\succ \omega_{i}} \sqcup\left\{\eta \backslash \omega_{i}: \eta \supsetneq \omega_{i}, \eta \succ \omega_{i}\right\},
$$




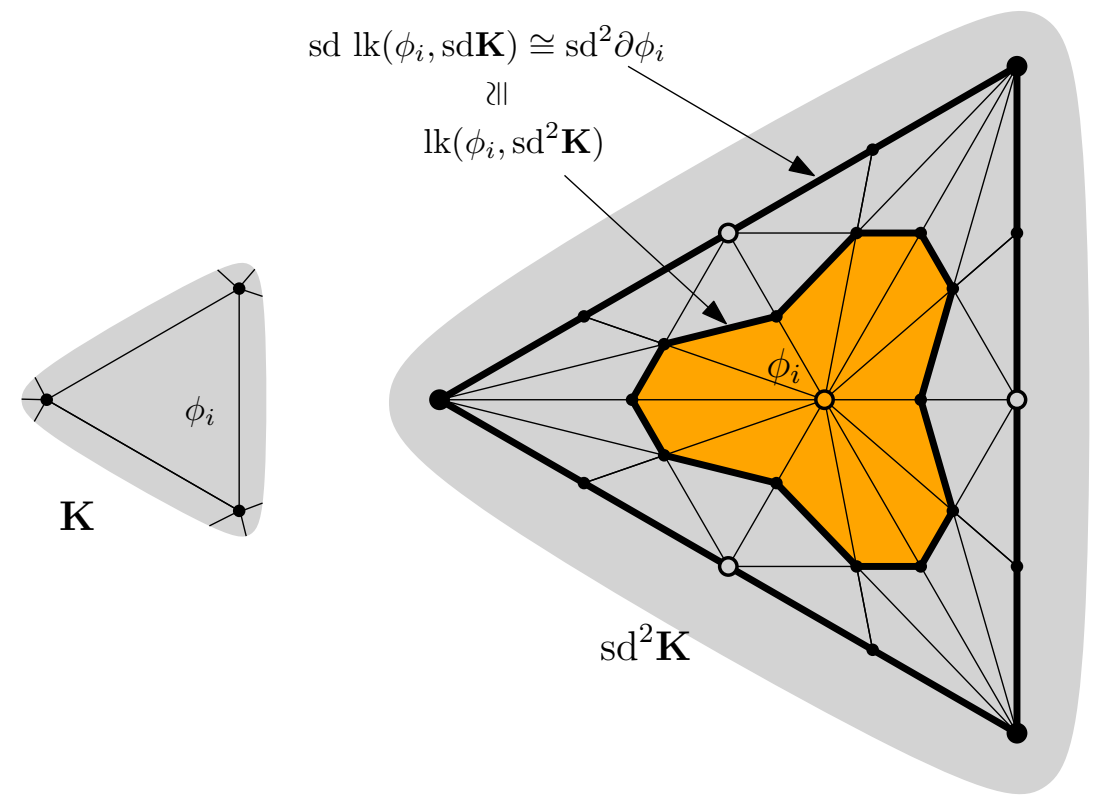

Figure 11: Isomorphisms for verifying the link condition in case 1. We consider the case of the removal of the facet $\phi_{i}$. If we were checking star decomposability only, we would be interested in star decomposability of $\mathrm{lk}\left(\phi_{i}, \mathrm{sd}^{2} \mathbf{K}\right)$. For star decomposability in vertices, this translates to checking the link condition for $\operatorname{sd} \operatorname{lk}\left(\phi_{i}, \mathrm{sd} \mathbf{K}\right)$ which is further isomorphic to $\operatorname{sd}^{2} \partial \phi_{i}$ (in this case, the last isomorphism is even equality).

which we denote by $W$. Thus, we need to check the star decomposability in vertices of the pair

$$
\left(\operatorname{sd}\left(\operatorname{sd} \partial \omega_{i} * \operatorname{sdlk}\left(\omega_{i}, \mathbf{K}\right)\right), W\right) .
$$

We distinguish several cases according to the type of $\omega_{i}$.

1. $\omega_{i}=\phi_{i}$, that is, $i \leq t$ :

In this case, $\phi_{i}$ is a facet. Therefore, $\operatorname{lk}\left(\phi_{i}, \mathbf{K}\right)=\emptyset$. Also $\eta \succ \phi_{i}$ for all proper subfaces $\eta$. Therefore, the pair (9) simplifies to $\left(\operatorname{sd}\left(\operatorname{sd} \partial \phi_{i}\right), V\left(\operatorname{sd} \partial \phi_{i}\right)\right)$; see Figure 11. That is, we only need that $\operatorname{sd}\left(\operatorname{sd} \partial \phi_{i}\right)$ is star decomposable in vertices which follows by the induction and Observation 16 .

2. $\omega_{i}=\sigma_{j}$ for some $j$, that is, $i>t$ and $t-i$ is odd:

We need to describe $W$, for which we need to describe the faces $\eta$ such that $\eta \subsetneq \sigma_{j}$ or $\sigma_{j} \subsetneq \eta$ such that $\eta \succ \sigma_{j}$. As $\sigma_{j}$ induces an elementary collapse in a sequence of collapses of $\mathbf{K}^{\prime}$, we get $\tau_{j} \succ \sigma_{j}$ but $\eta \prec \sigma_{j}$ for any $\eta \supsetneq \sigma_{j}$ different from $\tau_{j}$. On the other hand all proper subfaces of $\sigma_{j}$ are removed only later on in collapsing of $\mathbf{K}^{\prime}$. Altogether $W=V\left(\operatorname{sd} \partial \sigma_{j}\right) \sqcup\left\{\tau_{j} \backslash \sigma_{j}\right\}$. See Figure 12 for an example of the pair 9 in this case.

Now, we aim to use Corollary 13 with

$$
(\mathbf{X}, X)=\left(\operatorname{sd} \partial \sigma_{j}, V\left(\operatorname{sd} \partial \sigma_{j}\right)\right)
$$

and

$$
(\mathbf{Y}, Y)=\left(\operatorname{sdlk}\left(\sigma_{j}, \mathbf{K}\right),\left\{\tau_{j} \backslash \sigma_{j}\right\}\right)
$$




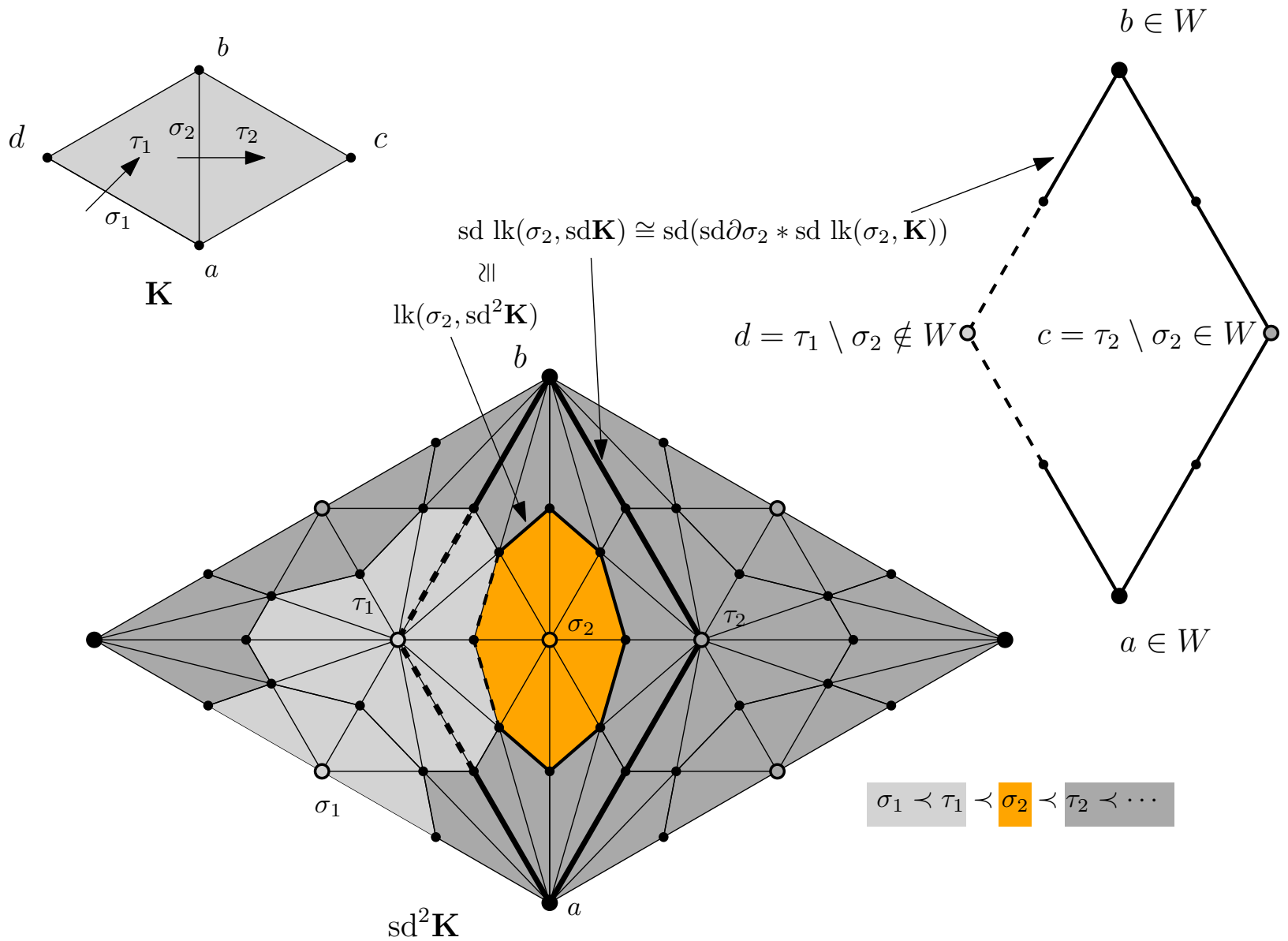

Figure 12: Isomorphisms for verifying the link condition in case 2. Here we consider the case $\sigma_{j}=\sigma_{2}$ coming from the collapses on the top left picture. The vertex decomposability of $\left(\operatorname{sdlk}\left(\sigma_{2}, \operatorname{sd} \mathbf{K}\right), V\left(\operatorname{lk}\left(\sigma_{2}, \operatorname{sd} \mathbf{K}\right)\right)_{\succ \sigma_{2}}\right)=\left(\operatorname{sdlk}\left(\sigma_{2}, \operatorname{sd} \mathbf{K}\right),\left\{a, b, \tau_{2}\right\}\right)$ in the middle picture translates to vertex decomposability of the pair $\left(\operatorname{sd}\left(\operatorname{sd} \partial \sigma_{2} * \operatorname{sd} l \mathrm{k}\left(\sigma_{2}, \mathbf{K}\right)\right), W\right)$ in the top right picture where $W=\left\{a, b, \tau_{2} \backslash \sigma_{2}\right\}$, which coincides with $V\left(\operatorname{sd} \partial \sigma_{j}\right) \sqcup\left\{\tau_{j} \backslash \sigma_{j}\right\}$ as required.

The pair $(\operatorname{sd} \mathbf{X}, X)$ is star decomposable in vertices by Observation 16 and the induction. For checking star decomposability in vertices of $(\operatorname{sd} \mathbf{Y}, Y)$, we know that $\operatorname{lk}\left(\sigma_{j}, \mathbf{K}\right)$ satisfies the (HRC) condition. In particular, $\operatorname{lk}\left(\sigma_{j}, \mathbf{K}\right)$ is collapsible after removing some number of facets, and the subsequent collapses can be rearranged so that the vertex $\tau_{j} \backslash \sigma_{j}$ is the last vertex in the sequence of collapses. (If $\operatorname{dim} \operatorname{lk}\left(\sigma_{j}, \mathbf{K}\right)=0$, then we instead rearrange the removals of the facets so that $\tau_{j} \backslash \sigma_{j}$ is the last.) Now, by induction, this sequence of removals of facets and collapses induces a star decomposition in vertices of $\operatorname{sd} \operatorname{sdlk}\left(\sigma_{j}, \mathbf{K}\right)$ such that $\left\{\tau_{j} \backslash \sigma_{j}\right\}$ is the last vertex in this decomposition. This exactly means that $(\mathrm{sd} \mathbf{Y}, Y)$ is star decomposable in vertices.

Altogether, Corollary 13 implies that the pair $(\operatorname{sd}(\mathbf{X} * \mathbf{Y}), X \sqcup Y)$ is star decomposable in vertices which is exactly the required pair (9).

3. $\omega_{i}=\tau_{j}$ for some $j$, that is, $i>t$ and $t-i$ is even:

We again first determine $W$. For each $\eta \supsetneq \tau_{j}$, we get $\eta \prec \tau_{j}$ as $\tau_{j}$ is a maximal face during the elementary collapse. On the other hand, for $\eta \subsetneq \tau_{j}$ we get $\eta \succ \tau_{j}$ unless $\eta=\sigma_{j}$ as all 


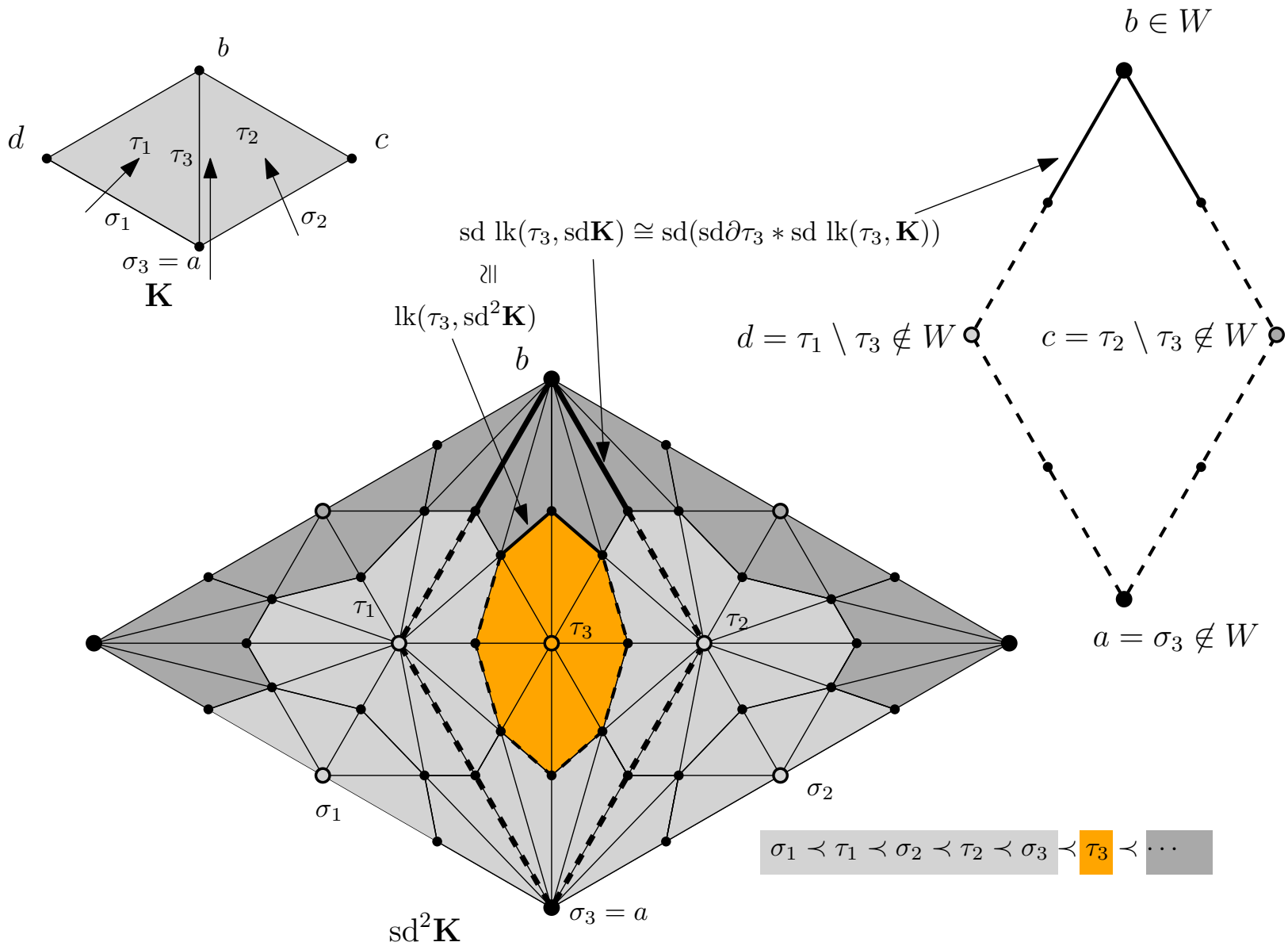

Figure 13: Isomorphisms for verifying the link condition in case 3. Here we consider the case $\tau_{j}=\tau_{3}$ coming from the collapses on the top left picture. The vertex decomposability of $\left(\operatorname{sdlk}\left(\tau_{3}, \operatorname{sd} \mathbf{K}\right), V\left(\operatorname{lk}\left(\tau_{3}, \operatorname{sd} \mathbf{K}\right)\right)_{\succ \tau_{3}}\right)=\left(\operatorname{sdlk}\left(\tau_{3}, \operatorname{sd} \mathbf{K}\right),\{b\}\right)$ in the middle picture translates to vertex decomposability of the pair $\left(\operatorname{sd}\left(\operatorname{sd} \partial \tau_{3} * \operatorname{sd} \operatorname{lk}\left(\tau_{3}, \mathbf{K}\right)\right), W\right)$ in the top right picture where $W=\{b\}$, which coincides with $V\left(\operatorname{sd} \partial \tau_{j}\right) \backslash\left\{\sigma_{j}\right\}$ as required.

subfaces of $\tau_{j}$ have to be present at the moment of removing of $\sigma_{j}$, and $\tau_{j}$ immediately succeeds. Altogether, $W=\left(V\left(\partial \tau_{j}\right) \backslash\left\{\sigma_{j}\right\}\right) \sqcup \emptyset$. See Figure 13 for an example of the pair (9) in this case.

We aim to use Proposition 14 with $\mathbf{X}=\operatorname{sdlk}\left(\tau_{j}, \mathbf{K}\right), \mathbf{Y}=\operatorname{sd} \partial \tau_{j}$ and $Y=V\left(\operatorname{sd} \partial \tau_{j}\right) \backslash\left\{\sigma_{j}\right\}$. We get that $\mathbf{X}$ is star decomposable in vertices by induction as $\operatorname{lk}\left(\tau_{j}, \mathbf{K}\right)$ satisfies the (HRC) condition. We also need that $(\operatorname{sd} \mathbf{Y}, Y)$ is star decomposable in vertices. For this we use Observation 16 and the induction while choosing $\sigma_{j}$ to be the first face removed from $V\left(\operatorname{sd} \partial \tau_{j}\right)$. Then $Y=V\left(\operatorname{sd} \partial \tau_{j}\right)_{\succ^{\prime}\left\{\sigma_{j}\right\}}$ where $\succ^{\prime}$ is the corresponding order on $V\left(\operatorname{sd} \partial \tau_{j}\right)$. Altogether, for application of Proposition 14 we choose the order $\succ^{\prime}$ on $V\left(\operatorname{sdlk}\left(\tau_{j}, \mathbf{K}\right)\right) \sqcup V\left(\operatorname{sd} \partial \tau_{j}\right)$ so that it starts with $\sigma_{j}$, it continues on $V\left(\operatorname{sdlk}\left(\tau_{j}, \mathbf{K}\right)\right.$ in order of a star decomposition in vertices of $\operatorname{sd} \mathbf{X}$ and finally it continues on $Y=$ $V\left(\operatorname{sd} \partial \tau_{j}\right) \backslash\left\{\sigma_{j}\right\}$ in the already prescribed order $\succ^{\prime}$. Then we get the required conclusion that $(\operatorname{sd}(\mathbf{X} * \mathbf{Y}), Y)$, which is the pair (9), is star decomposable in vertices. This finishes the proof of Theorem 2 . 


\section{Acknowledgments}

We thank to two anonymous referees for providing very useful comments.

\section{References}

[AB17] K. A. Adiprasito and B. Benedetti. Subdivisions, shellability, and collapsibility of products. Combinatorica, 37(1):1-30, 2017.

[BL13] B. Benedetti and F. H. Lutz. Knots in collapsible and non-collapsible balls. Electron. J. Combin., 20(3):Paper 31, 29, 2013.

[BW83] A. Björner and M. Wachs. On lexicographically shellable posets. Trans. Amer. Math. Soc., 277(1):323-341, 1983.

$\left[\mathrm{GPP}^{+} 19\right]$ X. Goaoc, P. Paták, Z. Patáková, M. Tancer, and U. Wagner. Shellability is NPcomplete. J. ACM, 66(3):21:1-21:18, 2019.

[Hac08] M. Hachimori. Decompositions of two-dimensional simplicial complexes. Discrete Math., 308(11):2307-2312, 2008.

[HT02] J. Herzog and Y. Takayama. Resolutions by mapping cones. Homology Homotopy Appl., 4(2, part 2):277-294, 2002. The Roos Festschrift volume, 2.

[Koz97] D. N. Kozlov. General lexicographic shellability and orbit arrangements. Ann. Comb., 1(1):67-90, 1997.

[Koz08] D. Kozlov. Combinatorial algebraic topology, volume 21 of Algorithms and Computation in Mathematics. Springer, Berlin, 2008.

[Lic91] W. B. R. Lickorish. Unshellable triangulations of spheres. European J. Combin., 12(6):527-530, 1991.

[PB80] J. S. Provan and L. J. Billera. Decompositions of simplicial complexes related to diameters of convex polyhedra. Math. Oper. Res., 5(4):576-594, 1980.

[RS82] C. P. Rourke and B. J. Sanderson. Introduction to piecewise-linear topology. Springer Study Edition. Springer-Verlag, Berlin-New York, 1982. Reprint.

[Whi39] J. H. C. Whitehead. Simplicial spaces, nuclei and m-groups. Proc. London Math. Soc. (2), 45(4):243-327, 1939. 